\title{
MKRN3 inhibits the reproductive axis through actions in kisspeptin-expressing neurons
}

\author{
Ana Paula Abreu, ${ }^{1}$ Carlos A. Toro, ${ }^{2}$ Yong Bhum Song, ${ }^{1}$ Victor M. Navarro, ${ }^{1}$ Martha A. Bosch, ${ }^{3}$ Aysegul Eren, ${ }^{1}$ Joy N. Liang, ${ }^{1}$ \\ Rona S. Carroll, ${ }^{1}$ Ana Claudia Latronico, ${ }^{4}$ Oline K. Rønnekleiv, ${ }^{3}$ Carlos F. Aylwin, ${ }^{2}$ Alejandro Lomniczi, ${ }^{2}$ Sergio Ojeda, ${ }^{2}$ \\ and Ursula B. Kaiser ${ }^{1}$
}

'Division of Endocrinology, Diabetes and Hypertension, Brigham and Women's Hospital and Harvard Medical School, Boston, Massachusetts, USA. ${ }^{2}$ Division of Neuroscience, Oregon National Primate Research Center-OHSU, Hillsboro, Oregon, USA. ²Department of Chemical Physiology and Biochemistry, Oregon Health and Science University, Portland, Oregon, USA. "Laboratório de Hormônios e Genética Molecular, Unidade de Endocrinologia do Desenvolvimento, Disciplina de Endocrinologia e Metabologia, Hospital das Clínicas, Faculdade de Medicina da Universidade de São Paulo, São Paulo, Brazil.

\begin{abstract}
The identification of loss-of-function mutations in $M K R N 3$ in patients with central precocious puberty in association with the decrease in MKRN3 expression in the medial basal hypothalamus of mice before the initiation of reproductive maturation suggests that MKRN3 is acting as a brake on gonadotropin-releasing hormone (GnRH) secretion during childhood. In the current study, we investigated the mechanism by which MKRN3 prevents premature manifestation of the pubertal process. We showed that, as in mice, MKRN3 expression is high in the hypothalamus of rats and nonhuman primates early in life, decreases as puberty approaches, and is independent of sex steroid hormones. We demonstrated that Mkrn3 is expressed in Kiss1 neurons of the mouse hypothalamic arcuate nucleus and that MKRN3 repressed promoter activity of human KISS1 and TAC3, 2 key stimulators of GnRH secretion. We further showed that MKRN3 has ubiquitinase activity, that this activity is reduced by MKRN3 mutations affecting the RING finger domain, and that these mutations compromised the ability of MKRN3 to repress KISS1 and TAC3 promoter activity. These results indicate that MKRN3 acts to prevent puberty initiation, at least in part, by repressing KISS1 and TAC3 transcription and that this action may involve an MKRN3-directed ubiquitinationmediated mechanism.
\end{abstract}

\section{Introduction}

The identification of loss-of-function mutations in MKRN3, encoding makorin ring finger protein 3 , in families with central precocious puberty (CPP) linked MKRN3 to the hypothalamic-pituitary-gonadal (HPG) axis for the first time (1). MKRN3 is a maternally imprinted gene located on chromosome 15q11.2, in the Prader-Willi syndrome (PWS) critical region, and was first identified and cloned during studies of PWS (2). Studies in humans and mice have determined that the MKRN3 maternal allele is methylated in the central nervous system (3). Thus, loss-of-function mutations affecting MKRN3 are paternally inherited (4). Although MKRN3 is ubiquitously expressed (3), loss of its actions in the hypothalamus is likely to significantly contribute to the premature initiation of puberty observed in patients with inactivating mutations of the gene $(1,4)$.

\section{Delated Commentary: p. 3957}

Authorship note: SO and UBK are co-senior authors and contributed equally to this work.

Conflict of interest: UBK has ownership of equity greater than $\$ 10,000$ in Amazon Amgen, Apple, Blueprint, CANbridge, Cigna, Cisco Systems, Express Scripts, Glycomimetics, Google, Hologic, Idera, ImmunoGen, Intel, Johnson \& Johnson, Microsoft Oracle, and Pfizer; income from the Endocrine Society, Aytu BioScience, Apnimed, Blueprint, Glycomimetics, Idera, and Immunogen (spouse); and research support from Ferring Research Institute.

Copyright: @ 2020, American Society for Clinical Investigation.

Submitted: January 23, 2020; Accepted: April 30, 2020; Published: July 20, 2020.

Reference information: J Clin Invest. 2020;130(8):4486-4500.

https://doi.org/10.1172/JCl136564.
Puberty is a complex biological process involving sexual maturation and, in humans, accelerated linear growth (5). Gonadotropin-releasing hormone $(\mathrm{GnRH})$ is produced in the hypothalamus and released in a coordinated pulsatile manner to stimulate the secretion of gonadotropins from the pituitary, which in turn act on the gonads to promote gametogenesis and the production of sex steroids (6). In humans, the HPG axis is active during the embryonic and neonatal stages of human life, becoming quiescent during childhood (7). The re-activation of the HPG axis is marked by an increase in amplitude and frequency of GnRH pulses, culminating in the phenotypic changes of puberty (7). Early re-activation of the HPG axis results in gonadotropin-dependent CPP, clinically defined as the development of secondary sexual characteristics before the age of 8 years in girls and 9 years in boys (8).

The factors regulating the complex pattern of $\mathrm{GnRH}$ secretion, and therefore puberty initiation, are not completely known. It is thought that pubertal timing is influenced by a tightly orchestrated interaction of genetic, nutritional, environmental, and socioeconomic factors (9). However, in some cases there appears to be a definitive component determining pubertal timing, as is the case in CPP associated with mutations in $M K R N 3$. After the first report linking MKRN3 to CPP, several other groups identified patients with CPP and mutations in $M K R N 3$, validating the association (10). To date, inactivating $M K R N 3$ mutations are the most common genetic defect associated with CPP (10). MKRN3 is the first protein to be identified that likely has an inhibitory role in GnRH secretion and puberty with mutations identified in humans. 

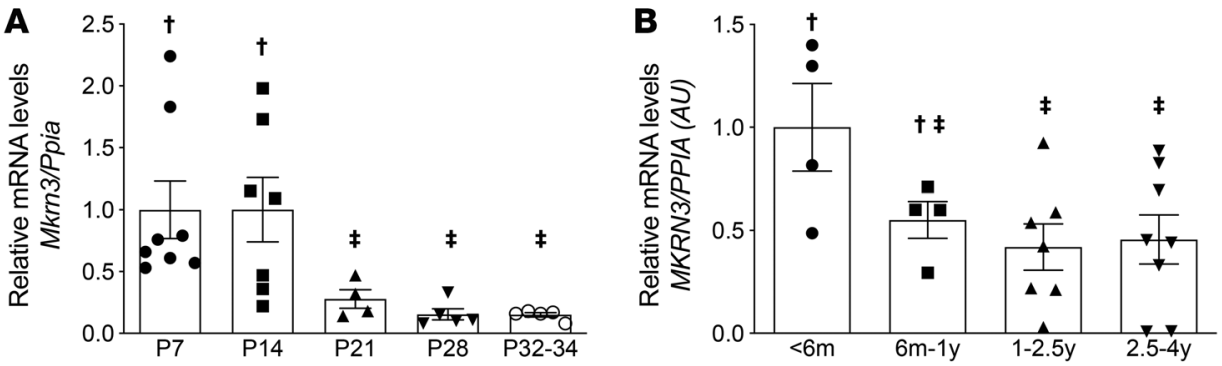

Figure 1. MKRN3 mRNA levels in the mediobasal hypothalamus (MBH) decrease before puberty initiation in different species. Expression of $M k r n 3$ mRNA in the hypothalamus of the female rat (A) or rhesus monkey (B) as determined by RT-qPCR. RNA expression data were normalized using peptidylproIyl isomerase A (Ppia) as the housekeeping gene and by dividing each individual value by the average of the P7 group for rats or the $<6 \mathrm{~m}$ group for monkeys. Bars represent mean \pm SEM. In A, groups are as follows: 7 days old $(P 7 ; n=8), 14$ days old (P14; $n=7), 21$ days old (P21; $n=4), 28$ days old (P28; $n=5)$, and 32-34 days old (P32-34; $n=5)$. In B, groups are as follows: monkeys younger than 6 months of age $(<6 \mathrm{~m} ; n=4), 6$ months to 1 year of age ( $6 \mathrm{~m}-1 \mathrm{y} ; n=4), 1$ to 2.5 years of age (1-2.5y; $n=7), 2.5$ to 4 years of age (2.5-4y; $n=8)$. Mkrn3 mRNA levels decreased from P14 to P21 in the hypothalamus of rats $(\mathbf{A})$, and from age $6 \mathrm{~m}$-1y to age $1.5-2 \mathrm{y}$ in the hypothalamus of monkeys (B). Groups with different symbols ( $\dagger$, $\ddagger)$ are significantly different $(\mathbf{A}, P=0.001 ; \mathbf{B}, P=0.046)$, as determined by 1-way ANOVA followed by the Student-Newman-Keuls test.

The mechanism by which MKRN3 deficiency leads to premature re-activation of GnRH secretion has not been elucidated. MKRN3 belongs to a family of E3 ubiquitin ligases; it has a centrally located C3HC4 RING finger motif, $3 \mathrm{CH} 3$ zinc finger motifs spaced along the length of the protein, and a motif with a unique pattern of conserved Cys-His residues called the makorin zinc finger (3). MKRN3 is a ubiquitous protein, abundantly expressed in the developing central nervous system of a wide array of eukaryotes (11). The findings that Mkrn3 expression decreases substantially in the medial basal hypothalamus $(\mathrm{MBH})$ of mice at the initiation of juvenile development (1) and the identification of loss-of-function mutations in patients with CPP (10) suggest that MKRN3 is acting as a brake on GnRH secretion during childhood (12). As such, MKRN3 appears to be an important component of the inhibitory apparatus that maintains GnRH in check before puberty (4). The prepubertal loss of Mkrn 3 expression observed in the MBH of juvenile male and female mice (1) implicates this region as a site where MKRN3 may exert its inhibitory effect on GnRH secretion. The arcuate nucleus (ARC), or infundibular region, which is contained within the $\mathrm{MBH}$, plays a key role in controlling puberty, and exerts this effect via neurons producing 2 key GnRH secretagogues, kisspeptin (encoded by KISS1/Kiss1) and neurokinin B (NKB, encoded by TAC3/Tac3) (13).

In the current study, we aimed to investigate the mechanism by which MKRN3 prevents the premature manifestation of the pubertal process. We first characterized the expression pattern of $M k r n 3$ mRNA in the brain and validated in rats and nonhuman primates the prepubertal decrease in hypothalamic Mkrn3 expression previously observed in mice. We showed that this decrease occurs independently of gonadal activation, and identified Kiss1 mRNA-containing neurons of the ARC as a population of hypothalamic cells expressing Mkrn3. Our results also demonstrated that MKRN3 associates with the KISS1 and TAC3 gene promoters to repress transcriptional activity, and that this inhibitory effect is prevented by MKRN3 mutations identified in the RING finger domain in patients with CPP, which compromise MKRN3-dependent ubiquitinase activity.

\section{Results}

Prepubertal loss of hypothalamic Mkrn3 expression occurs in both rodents and nonhuman primates. We previously showed higher Mkrn $3 \mathrm{mRNA}$ levels in the ARC of male and female mice at postnatal day 10 (P10), with a striking reduction from P12 to P22 that persisted into adulthood (1). To assess the developmental profile of Mkrn3 mRNA in the $\mathrm{MBH}$ of another rodent model, we examined the changes in $M k r n 3$ expression that occur during postnatal development of female rats by reverse transcription real-time quantitative PCR (RT-qPCR). In agreement with the previous mouse studies (1), Mkrn3 mRNA levels were high in the MBH at P7 and P14, decreasing strikingly by P21 and remaining low thereafter $(P=0.001$; ANOVA followed by the Student-Newman-Keuls test) (Figure 1A). A similar profile was observed in the MBH of female rhesus monkeys, with MKRN3 mRNA levels decreasing after postnatal age 1 year and remaining low thereafter $(P=0.046)$ (Figure 1B). These results indicate that the prepubertal decrease in hypothalamic MKRN3 expression is not exclusive to mice, as it is also observed in rats and in nonhuman primates.

The prepubertal decrease in hypothalamic Mkrn3 is independent of gonadal activation. To determine whether the prepubertal decrease in hypothalamic Mkrn3 expression occurs independently of gonadal function, we measured Mkrn3 mRNA levels in the ARC of wild-type (WT) and hypogonadal $(h p g)$ male and female mice across development. Hpg mice have a deletion in Gnrh1, leading to a lack of GnRH secretion, absence of pubertal development, and infertility (14). We also measured Mkrn3 expression in the anteroventral periventricular nucleus (AVPV) of these mice, a region harboring neuronal networks (most prominently Kiss1 neurons) required for the luteinizing hormone surge and the completion of pubertal maturation in female rodents (15).

Similarly to WT mice, Mkrn3 mRNA levels in $h p g$ mice decreased in the ARC across postnatal maturation, becoming significantly lower by P30 compared with P10 $(P=0.0002$; ANOVA, Student-Neuman-Keuls post hoc test) in both female and male mice (Figure 2, A and B), and remaining at these lower levels thereafter $(P<0.0002)$; ANOVA, Tukey's multiple-comparisons post hoc test). Additionally, Mkrn3 mRNA levels were not statistically different (by 1-way ANOVA) in $h p g$ and age-matched WT 

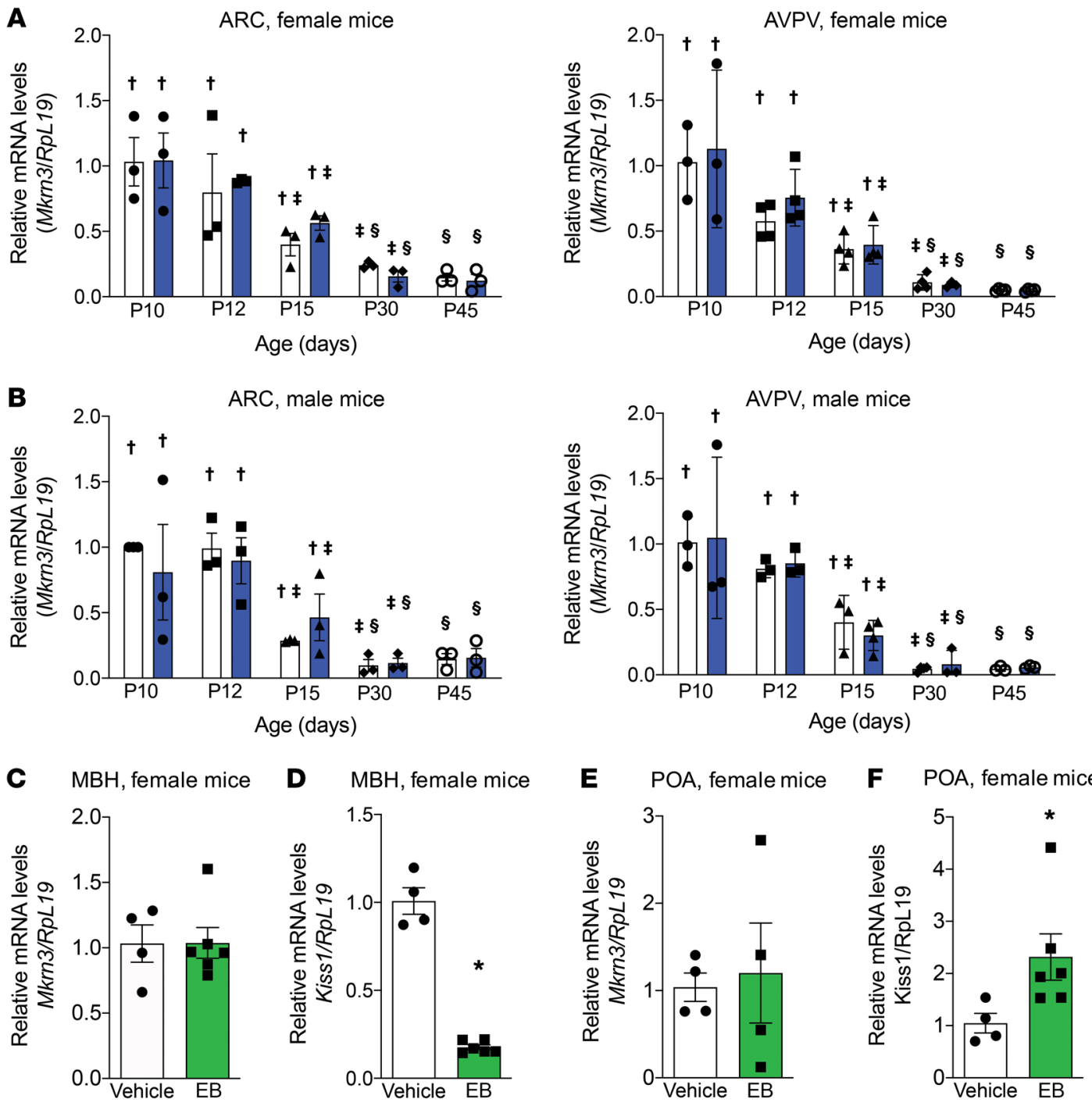

Figure 2. The prepubertal decrease in hypothalamic Mkrn3 expression is independent of gonadal activation. Mkrn3 expression in the ARC (left) and AVPV (right) of intact WT (white bars) and hpg (blue bars) mice across postnatal development as determined by RT-qPCR. The bar graphs show the relative change in mRNA levels in female (A) and male (B) mice, compared with levels at P10, normalized to levels of endogenous ribosomal protein L19 (RpL19) mRNA. Mean $( \pm$ SEM) values are shown at each age $(n=3-4$ mice per group, with each measurement performed in triplicate). Statistical analysis of effects of age and genotype were compared by 2-way ANOVA with a post hoc Tukey's multiple-comparisons test. Mkrn3 mRNA levels decreased from P10 to P45 in the ARC and AVPV of WT mice, similarly to hpg mice. There was no difference in Mkrn3 expression between WT and hpg female or male mice across pubertal maturation in either the ARC or AVPV. Groups with different symbols $(\dagger, \ddagger$, $)$ are significantly different $(P=0.0002)$. (C-F) Expression of Mkrn3 (C and E) and Kiss $1(\mathbf{D}$ and $\mathbf{F})$, quantified by RT-qPCR, in the MBH (C and $\mathbf{D})$ and POA (E and $\mathbf{F}$ ) of WT female mice treated with $2 \mu \mathrm{g}$ of estradiol benzoate (EB)or vehicle at age P11. All mice were sacrificed 24 hours after treatment. The bar graphs show relative mRNA levels in female EB-treated (green bars) mice compared with vehicle-treated (white bars) mice, normalized to levels of endogenous RpL19 mRNA. Mean ( \pm SEM) values are shown for 4-6 mice in each group, with each measurement performed in triplicate. Statistical analysis of effects of EB treatment was by unpaired 2-tailed $t$ test. Asterisks indicate $P<0.0001$ for $\mathbf{D}$ and $P=0.014$ for $\mathbf{F}$.

mice at all ages examined, suggesting that the absence of sex steroids in $h p g$ mice did not affect Mkrn3 expression.

Interestingly, Mkrn3 mRNA levels in the AVPV followed the same pattern as in the ARC, decreasing after P10 in both male and female WT and $h p g$ mice (Figure 2, A and B, $P=0.0002$; ANOVA). This age-dependent change suggests that any influence of $M k r n 3$ on AVPV function may decrease as puberty approaches, as previously suggested for the ARC.

To investigate more specifically the possibility of estradiol regulation of Mkrn3 expression, we treated WT female mice at P11 with $2 \mu$ g estradiol benzoate (EB) $(\sim 3 \mu \mathrm{g}$ per $10 \mathrm{~g}$ body weight) or vehicle (sesame oil) subcutaneously and measured Mkrn3 expression 24 hours later in the $\mathrm{MBH}$ and preoptic area (POA) region encompassing the AVPV. EB treatment did not alter Mkrn3 mRNA levels in either the $\mathrm{MBH}$ or the POA of these mice compared with vehicle-treated mice (Figure 2C). As a positive control for the action of EB, we measured Kiss1 expression in these regions. Kiss1 expression is positively regulated by estradiol in the AVPV of female mice and inhibited by estradiol in the $\operatorname{ARC}(15,16)$. In agreement with these known effects, Kiss1 expression decreased in the $\mathrm{MBH}$ and increased in the POA (Figure 2, D and F) following EB treatment $(P<0.0001$ for MBH; $P=0.014$ for $\mathrm{POA})$, compared with 
A

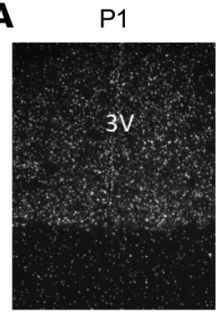

P10

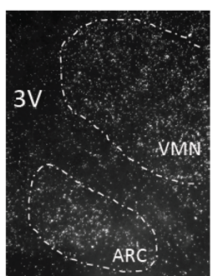

P15

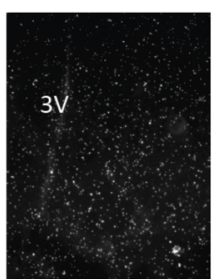

Adult OVX

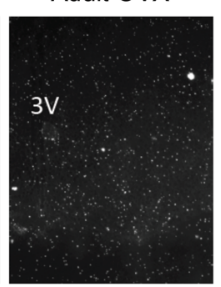

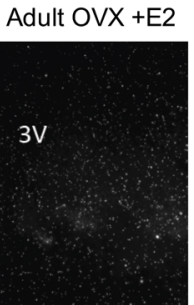

P1 (sense)

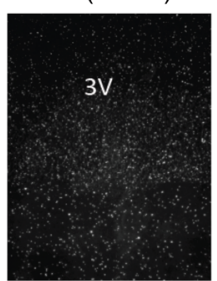

B Mkrn3 expression female mouse P10

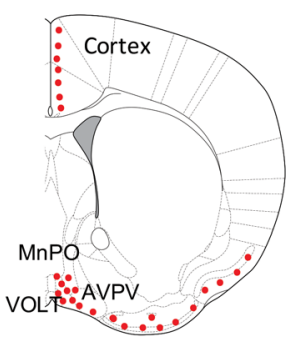

C

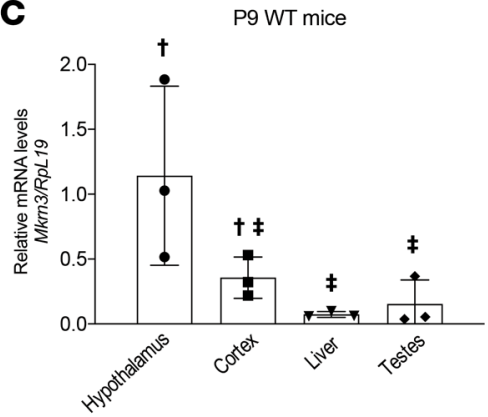

0 - 0 or

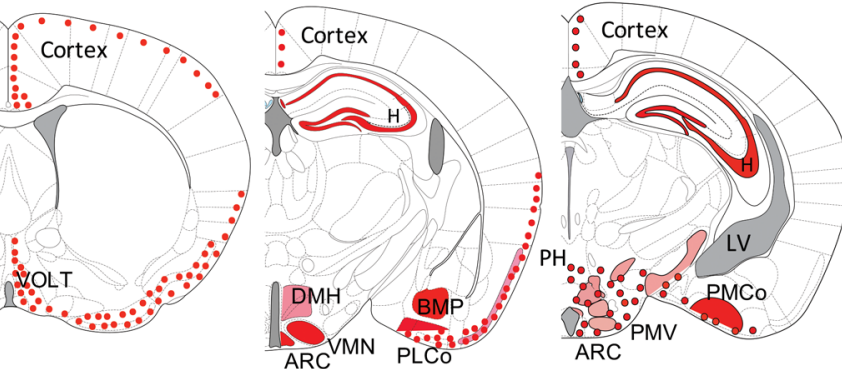

D

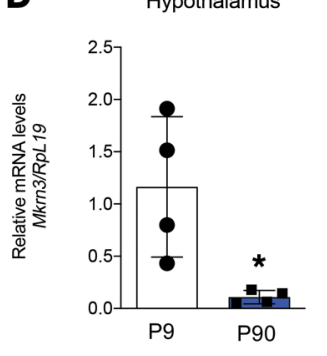

E

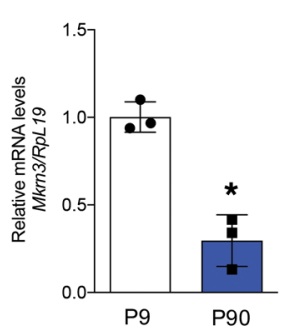

$\mathbf{F}$

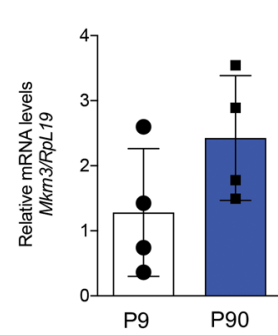

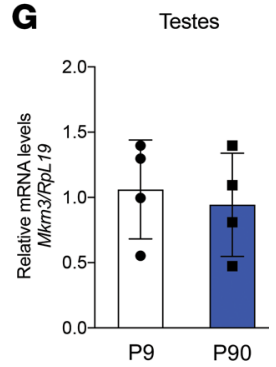

Figure 3. Mkrn3 expression is higher in the hypothalamus and cortex of mice compared with peripheral tissues before puberty onset. (A) Representative photomicrographs showing Mkrn3 expression by in situ hybridization (ISH) in female mice in the caudal hypothalamus. A high, diffuse level of Mkrn3 expression was detected in the hypothalamus of P1 mice, with more specific localization of expression in the ARC and VMN at P10. Expression was reduced by $\mathrm{P} 15$ and low in the adult in both ovariectomized (OVX) and estradiol-replaced (E2-replaced) mice. A sense probe did not detect Mkrn3 mRNA. $3 \mathrm{~V}$, third ventricle; ARC, arcuate nucleus; VMN, ventromedial nucleus. (B) Schematic representation of the neuroanatomical distribution of Mkrn3 mRNA in the brain of female P10 mice, as assessed by ISH. Red dots indicate areas where Mkrn3 mRNA was detected, darker areas with higher concentrations, and lighter red with relatively low Mkrn3 expression. MnPo, median preoptic nucleus; VOLT, vascular organ of the lamina terminalis; $\mathrm{H}$, hippocampus; DMH, dorsomedial nucleus of hypothalamus; PLCo, posterolateral cortical amygdaloid nucleus; BMP, accessory basal amygdaloid nucleus; PH, posterior hypothalamic area; PMV, premammillary nucleus, ventral part; PMCo, posteromedial cortical amygdaloid nucleus; LV, lateral ventricle. (C) Mkrn3 expression in tissues of P9 male mice. (D-G) Mkrn3 expression in the hypothalamus (D), cortex (E), liver (F), and testes (G) of mice at P9 (white bar) and P90 (blue bar). The bar graphs show the relative change in mRNA levels in different tissues, compared with Mkrn3 levels in the hypothalamus in C and with levels at Pg in D-G. Mean ( \pm SEM) values are shown at each age ( $n=3-4$ mice per group, with each measurement performed in triplicate). In C, groups with different symbols $(\dagger, \ddagger)$ are significantly different $(P=0.039)$, as determined by 1 -way ANOVA followed by Tukey's multiple-comparisons test. In $\mathbf{D}$ - $\mathbf{G}$, statistical analysis was performed by unpaired 2-tailed $t$ test. Asterisks indicate $P=0.002$ in $\mathbf{D}$ and $P=0.02$ in $\mathbf{E}$.

vehicle-treated controls, confirming that the EB treatment was effective. Taken together, these findings support the notion that regulation of Mkrn3 expression in the $\mathrm{MBH}$ and POA is a gonadal steroid-independent process.

High Mkrn3 expression in the hypothalamus during early postnatal life. To more comprehensively characterize the distribution of Mkrn3 mRNA in the mouse hypothalamus, we analyzed Mkrn3 mRNA expression by in situ hybridization (ISH) in mice at different postnatal ages (Figure 3A). A high, diffuse level of Mkrn3 expression was detected in the hypothalamus of P1 male (not shown) and female (Figure 3A) mice, with more specific localization of Mkrn3 expression in the ARC and ventromedial nucleus (VMN) of female mice by P10 (Figure 3A). Mkrn3 mRNA levels were mark- edly decreased by P15 and remained low in the adult, irrespective of the estrogen milieu (i.e., in both ovariectomized and estradiolreplaced mice) (Figure 3A), consistent with our RT-qPCR findings (Figure 2). Mkrn3 mRNA was absent in sections incubated with a sense-strand Mkrn3 probe (Figure 3A). Mkrn3 mRNA was also expressed in the cortex, hippocampus, lateral ventricle (LV), vascular organ of the lamina terminalis (VOLT), median preoptic nucleus (MnPo), AVPV, dorsomedial nucleus of hypothalamus (DMH), posterolateral cortical (PLCo) and posteromedial cortical (PMCo) and accessory basal (BMP) amygdaloid nuclei, premammillary nucleus, ventral part (PMV), and posterior hypothalamic area (PH) (Figure 3B). Within the hypothalamus, Mkrn3 was found to be most highly expressed in the ARC and VMN (Figure 3B). 

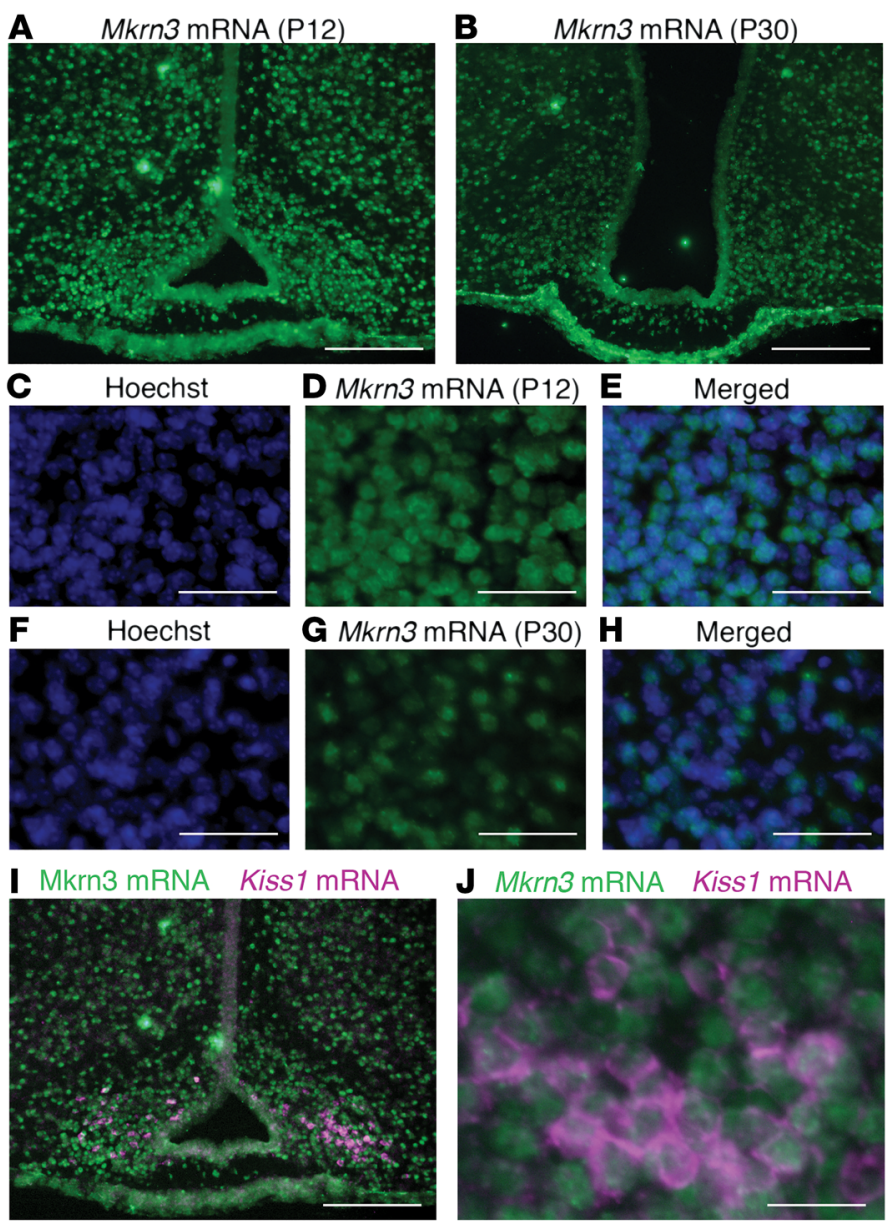

J Mkrn3 mRNA Kiss1 mRNA

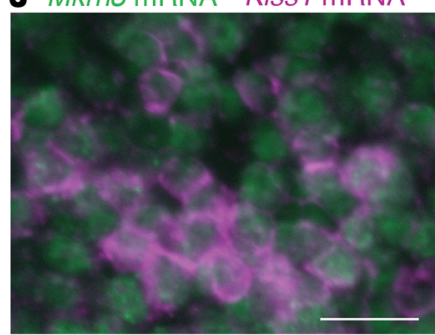

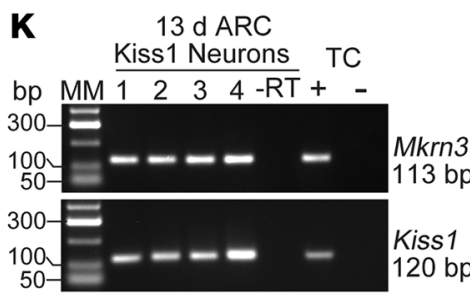

L Kiss1 ARC Neurons

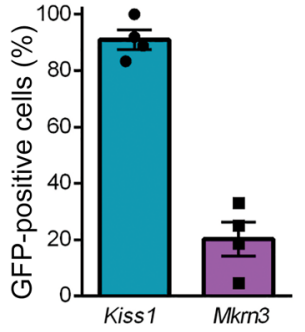

Figure 4. Mkrn3 is coexpressed in Kiss1 neurons in the MBH of mice. (A and B) Double-labeled fluorescent in situ hybridization (FISH) for Mkrn3 (green) and Kiss1 (magenta) mRNA in the MBH of P12 and P30 female mice. Mkrn3 mRNA was detected in several neurons in the ARC of P12 mice (A), with a decrease in P30 mice (B). Cell nuclei identified by Hoechst staining (blue) compared with Mkrn3 expression of (C-E) P12 and (F-H) P30 mice in higher-magnification views. (I and J) Neurons of P12 mice coexpress Mkrn3 and Kiss1 mRNA. Scale bars: $100 \mu \mathrm{m}$ (A and B) and $10 \mu \mathrm{m}$ (I-J and C-H). (K) Representative gel showing Mkrn3 and Kiss1 expression by RT-PCR in individual Kiss1 $1^{\text {CrecFP }}$ cells from the ARC in intact P13 females. The expected sizes for Mkrn3 and Kiss1 are 113 and 120 bp, respectively. RNA extracted from the MBH was used as positive (+, with reverse transcriptase) and negative (-, without reverse transcriptase) controls (TC, tissue control). (L) Bar graphs summarizing the percentage (mean \pm SEM) of Kiss $1^{\text {CrecFP }}$ neurons (73 neurons from 4 animals) that expressed Kiss1 and Mkrn3 mRNA.

Mkrn3 is expressed in several tissues in mice (2). To assess $M k r n 3$ expression in different mouse tissues, we analyzed Mkrn3 mRNA levels in the hypothalamus, cortex, liver, and testes of P9 and $\mathrm{P} 90$ mice. At P9, Mkrn3 mRNA levels were higher in the hypothalamus than in the liver and testes (Figure 3C), suggesting an important role of the gene in this region. While levels decreased significantly at $\mathrm{P} 90$ in the hypothalamus $(P=0.002)$ and cortex $(P$ $=0.02$ ) compared with $P 9$, levels remained unchanged by age in the liver and testes (Figure 3, D-G).

$M k r n 3$ is expressed in Kiss1 neurons in the mouse MBH. To determine if $M k r n 3$ is expressed in ARC kisspeptin-expressing neurons (Kiss1 neurons), we performed double-labeled fluorescent in situ hybridization (FISH) studies on the ARC of female mice at P12 and P30 and single-cell RT-PCR at P13. In keeping with our RT-qPCR and ISH results, the number of cells expressing Mkrn3 in the ARC decreased substantially between P12 and P30 (Figure 4, A and B). Although many cells contain Mkrn3 transcripts at P12, Hoechst staining demonstrated that Mkrn3 expression was not detected in all cells but rather limited to a subpopulation of cells (Figure 4, C-E), whose numbers decreased strikingly at P30 (Figure 4, F-H). Double-labeled FISH showed that most ARC Kiss1 neurons, identified by the presence of Kiss1 mRNA, also contained Mkrn3 transcripts at P12 (Figure 4, I and J).

Based on initial findings that Mkrn3 mRNA levels were the highest early postnatally but barely detectable in the POA and $\mathrm{MBH}$ of pubertal and adult female mice (Figures 1-4), we focused the single-cell analysis of Kiss1 neurons on 13-day-old mouse pups. Seventy-three Kiss1 $1^{\text {CreGFP }}$ neurons were harvested from the ARC of 13-day-old Kiss 1 CreGFP female mice ( $n=4$ animals); $91 \%$ expressed Kiss 1 mRNA, whereas Mkrn3 mRNA was detected in $25 \%$ of the neurons (Figure $4, \mathrm{~K}$ and $\mathrm{L}$ ).

MKRN3 selectively inhibits KISS1 and TAC3 promoter activity. The expression of Mkrn3 in Kiss1 neurons led us to hypothesize that MKRN3 may act by repressing the transcription of genes encoding GnRH stimulatory neuropeptides produced in these neurons, namely KISS1 and/or TAC3 (17-19). To test this hypothe- 

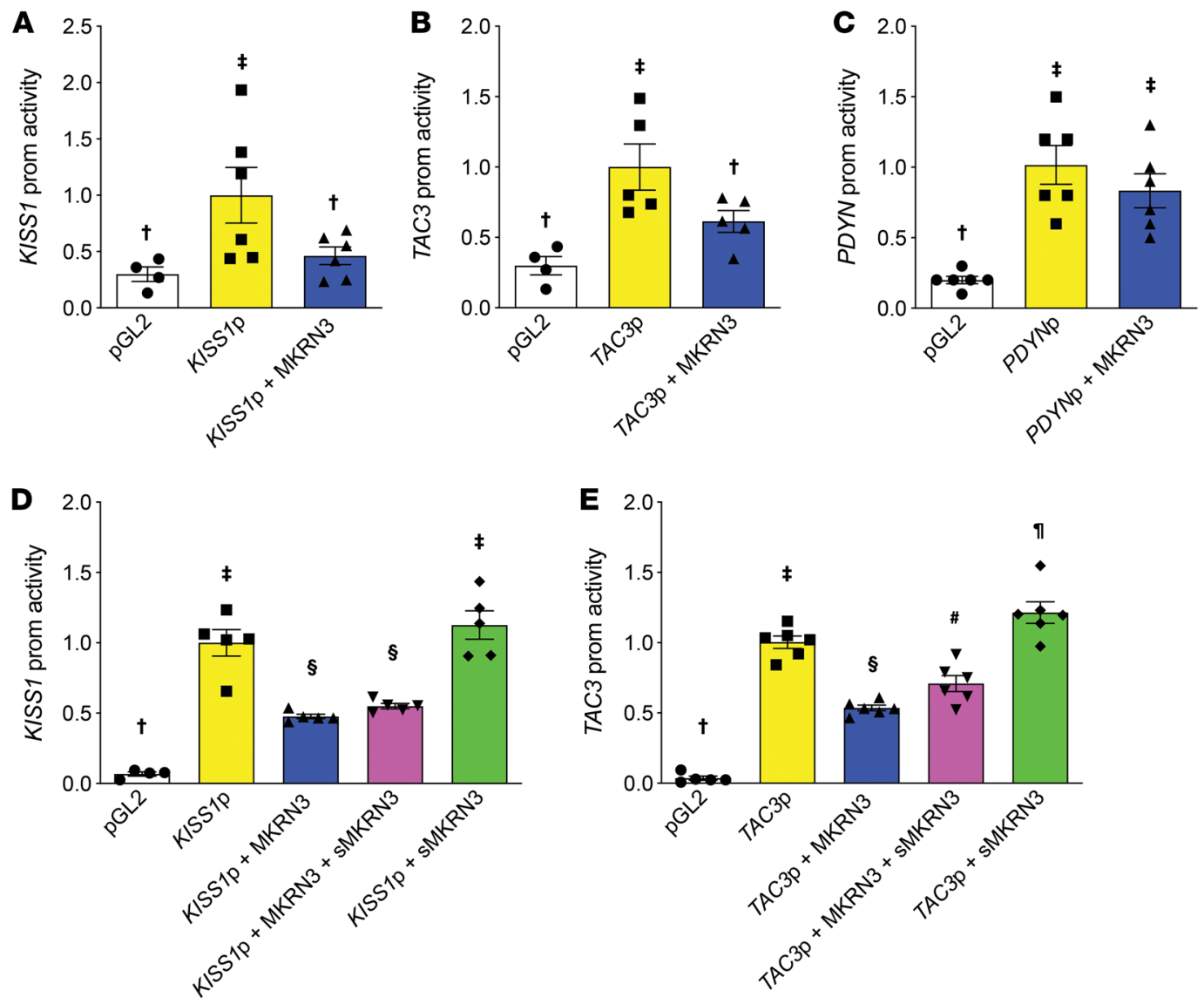

Figure 5. MKRN3 inhibits KISS1 and TAC3 promoter activity. Effect of MKRN3 on luciferase activity regulated by the human KISS1 (A), TAC3 (B), and PDYN (C) promoter (p). Effect of short truncated MKRN3 (sMKRN3) on KISS1 (D) and TAC3 (E) promoter activity. Neuro-2a cells were transfected with luciferase reporter constructs containing the $5^{\prime}$ flanking region of the indicated genes, in addition to an expression vector encoding WT MKRN3-HA or MKRN3 mutants. Forty-eight hours later, the cells were harvested and assayed for luciferase activity. Bars represent mean \pm SEM $(n=4-6)$. (A-C) Although WT MRKN3 inhibited KISS1 and TAC3 $(P=0.0056)$ promoter activity, it did not inhibit PDYN promoter activity. (D and E) A truncated MKRN3 (sMKRN3) with 102 amino acids did not inhibit KISS1 or TAC3 promoter activity. Yellow bars represent the indicated gene promoters only, blue bars represent the indicated gene promoters and cotransfection with MKRN3, pink when both MKRN3 and sMKRN3 were cotransfected, and green when only sMKRN3 was cotransfected. Groups with different symbols $(\dagger, \ddagger, \S, \#, \uparrow)$ are significantly different $(P<0.05)$, as determined by 1 -way ANOVA followed by the Student-Newman-Keuls test.

sis, we transfected Neuro-2a cells with a pGL2 luciferase vector in which luciferase expression is driven by either the human KISS1 or TAC3 promoter (20), with or without an expression vector encoding human MKRN3. Transfection of either promoter resulted in increased luciferase activity compared with the empty pGL2 vector, and this activity was significantly reduced $(\sim 40 \%-60 \%)$ by MKRN3 coexpression (Figure 5, A and B).

Next, we determined if MKRN3 was able to repress the promoter activity of other genes implicated in the stimulatory and inhibitory control of puberty. MKRN3 failed to repress transcription of EAP1 (Supplemental Figure 1; supplemental material available online with this article; https://doi.org/10.1172/JCI136564DS1), a transcription factor recently shown to increase GnRH expression and to carry loss-of-function mutations in individuals with self-limited delayed puberty (21). MKRN3 also failed to repress the promoter activity of other putative puberty-activating genes ( $T t f 1$ and Vglut2) (Supplemental Figure 1), and puberty-inhibitory genes (PDYN, Viaat1, and Eed) (Figure 5C and Supplemental Figure 1). These data indicate that MKRN3 selectively represses KISS1 and TAC3 promoter activity, suggesting that MKRN3 may act on Kiss1 neurons to reduce kisspeptin and NKB output, ultimately resulting in decreased GnRH secretion.

Most of the MKRN3 mutations identified in patients with CPP are frameshift mutations, resulting in truncated, inactive proteins (10). Using 3'-RACE PCR, we identified in human hypothalamic RNA a variant form of mRNA encoding a truncated form of MKRN3 that lacks the RING finger domain (Supplemental Figure 2). This truncated form (sMKRN3), with 102 amino acids, analogous to the truncated variants encoded by frameshift mutations in MRKN3, was unable to repress KISS1 and TAC3 promoter activity and did not prevent the repressive effect of full-length WT MKRN3 on these promoters (Figure 5, D and E).

MKRN3 missense mutations identified in patients with CPP are predicted to result in loss of function of MKRN3 based on in silico analysis and ab initio modeling $(22,23)$. We tested the effects of p.C340G, p.R365S, p.F417I, and p.H420Q mutant variants of MKRN3, identified in patients with CPP $(4,22-24)$, on the ability of MKRN3 to inhibit KISS1 and TAC3 promoter activity. The p.C340G mutation, which compromises the integrity of the RING finger domain by removing a key cysteine residue (Figure 6C), resulted 

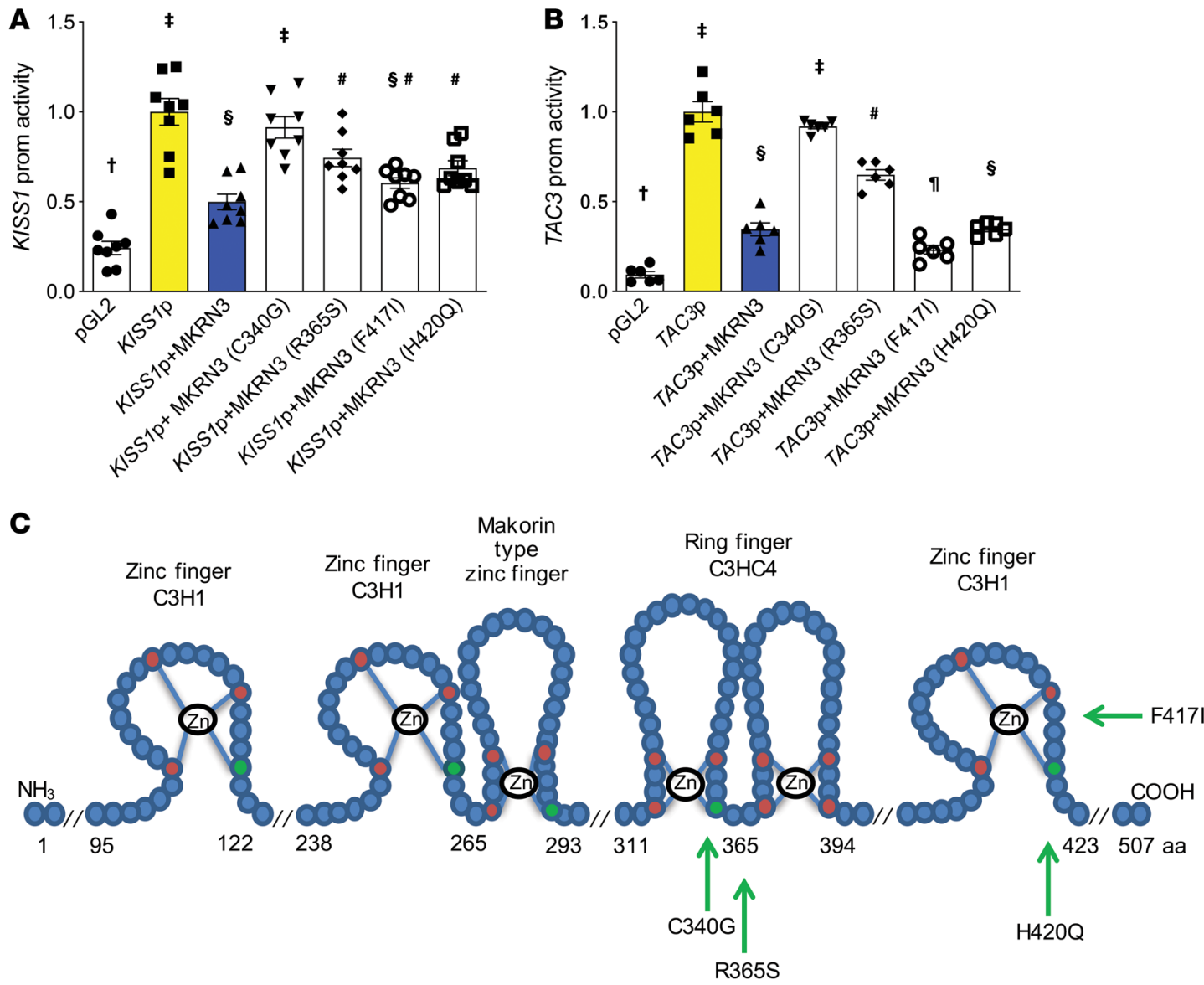

Figure 6. MKRN3 RING finger mutants lose the ability to inhibit KISS1 and TAC3 promoters. Effect of missense MKRN3 mutations identified in patients with CPP on KISS1 (A) and TAC3 (B) promoter activity. The mutants located in the RING finger domain, p.C340C and p.R365S, lost the ability to inhibit the KISS1 and TAC3 promoters, similarly to WT MKRN3. The mutant located in the zinc finger domain, p.F417I, inhibited the KISS1 and TAC3 promoters at least as effectively as WT MKRN3, while p.H420Q, located in the same domain as F417, inhibited the TAC3 promoter similarly to WT MKRN3 but had compromised ability to inhibit the KISS1 promoter. Yellow bars represent the KISS1 or TAC3 promoter, blue bars represent the KISS1 or TAC3 promoter with cotransfection of MKRN3. Groups with different symbols $(\dagger, \ddagger, \S, \#, q)$ are significantly different $(P<0.05)$, as determined by 1 -way ANOVA followed by Student-Newman-Keuls test. (C) Schematic representation of the makorin ring finger protein encoded by MKRN3 showing the zinc finger domains, which are RNA binding domains, the RING finger domain, responsible for E3 ubiquitin ligase activity, and the specific makorin-type domain. The arrows point to the locations of the missense mutations studied here. $\mathrm{Zn}$, zinc finger; pink circles, cysteine residues in the zinc fingers; green circles, histidine residues in the zinc fingers; blue circles represent the amino acids in the protein. The numbers underneath the schematic indicate amino acid locations in the protein.

in total loss of the inhibitory effect of MKRN3 on KISS1 and TAC3 promoter activity (Figure 6, A and B). The other mutation of the RING finger domain, p.R365S (Figure 6C), resulted in a significant, though incomplete, reduction in MKRN3 inhibitory activity on both promoters (Figure 6, A and B). In contrast, mutations affecting the $\mathrm{C}$-terminal zinc finger domain downstream from the RING finger domain (p.F417I and p.H420Q, Figure 6C), compromised MKRN3 inhibitory activity either partially (KISS1) or not at all (TAC3) (Figure 6, A and B). These findings indicate that only mutations affecting the RING finger domain, but not those affecting the downstream zinc finger domain, have substantial deleterious effects on MKRN3 transcription-repressive activity.

To determine if integrity of the RING finger domain and/or the C-terminal zinc finger domain are required for MKRN3 binding to the KISS1 and/or TAC3 promoters, we performed chromatin immunoprecipitation (ChIP) assays. The results showed that WT MKRN3 as well as the p.C340G, p.R365S, and p.H420Q mutants were recruited at the same rate to both the KISS1 and TAC3 pro- moters. In contrast, binding of the p.F417I mutant to both promoters was significantly reduced (Figure 7, A and B). These findings indicate that the RING finger domain is not essential for MKRN3 recruitment to either the KISS1 or the TAC3 promoter.

MKRN3 is a ubiquitin ligase. MKRN3 contains a C3HC4 RING finger motif characteristic of E3 ubiquitin ligases (4). We therefore sought to determine if MKRN3 is a ubiquitin ligase, and if this activity requires the integrity of the RING finger domain, the C-terminal zinc finger domain, or both. A notable feature of RING E3 ubiquitin ligases is that the enzymatic activity of the E3 ligase can be monitored through auto-ubiquitination of the protein in vitro (25). Auto-ubiquitination is the process by which the E3 enzymes catalyze the addition of polyubiquitin to themselves (26).

We took advantage of this property to assess MKRN3 ubiquitinating activity by determining if MKRN3 undergoes auto-ubiquitination by Western blot analysis of HEK293T cells cotransfected with a plasmid encoding MKRN3, and a plasmid encoding ubiquitin. Whole-cell lysates revealed a smear of larger complexes, typi- 
A

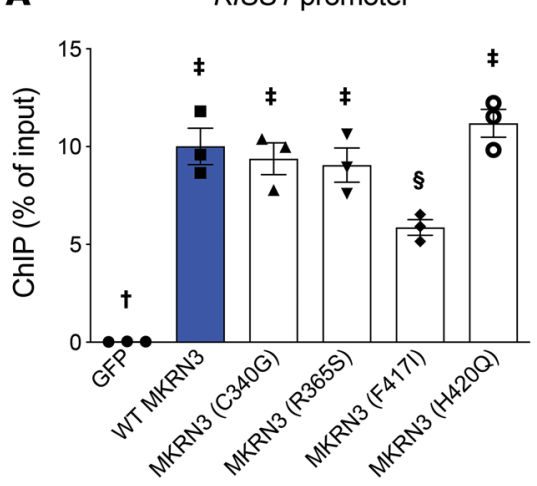

B

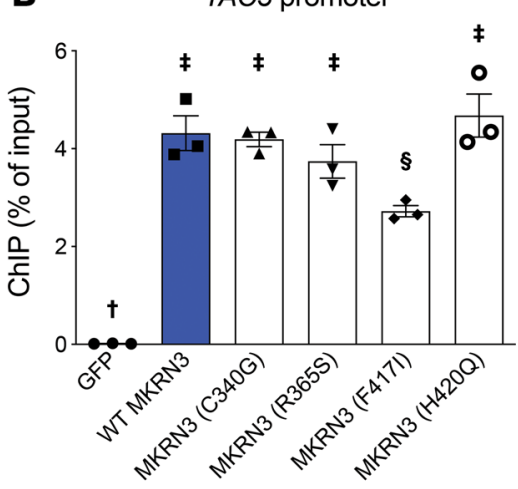

Figure 7. WT and mutant MKRN3 bind to KISS1 and TAC3 promoters. Association of WT MKRN3 and mutant variants, identified in patients with CPP, with the $5^{\prime}$ promoter region of the KISS1 (A) and TAC3 (B) genes as determined by chromatin immunoprecipitation assay. HEK293T cells were transfected with MKRN3 constructs carrying a $3 \times$ hemagglutinin (HA) tag (WT MKRN3 and mutants C340C, R365S, P417I, and H420Q), and the negative control was transfected with a GFP construct devoid of MKRN3. Immunoprecipitation was carried out using anti-HA antibodies. Bars represent mean \pm SEM $(n=3)$. Blue bars represent WT MRKN3. Groups with different symbols $(\dagger, \ddagger, \S)$ are significantly different from one another $(P<0.001)$, as determined by 1-way ANOVA followed by the Student-Newman-Keuls test. cal of the pattern shown by polyubiquitinated proteins, suggesting that MKRN3 is auto-ubiquitinated. No bands were detected from untransfected cells (Figure 8A).

Mutations in MKRN3 reduce cellular protein levels. To determine the effect of the missense mutations p.C340G, p.R365S, p.F417I, and p.H420Q identified in patients with CPP on MKRN3 protein levels and MKRN3-dependent ubiquitination, we transfected plasmids encoding HA-tagged WT or mutant MKRN3 into HEK293T cells. Western blot analysis of cell lysates using an anti-HA antibody showed that WT MKRN3 and all mutants were expressed at similar levels and displayed 2 bands (Figure 8, B and C). Lysates immunoprecipitated with the anti-HA antibody and then probed with the same anti-HA antibody showed similar patterns (Figure 8, B and C). However, in both cases the abundance of the $45-\mathrm{kDa}$ band was markedly reduced for the p.C340G mutant (Figure 8, B and C). Probing the blots with anti-ubiquitin antibodies showed that all mutants, particularly p.C340G and p.R365S, have a reduced signal compared with WT MKRN3, indicating that these mutations impair MKRN3 E3 ubiquitin ligase activity, even with similar levels of immunoprecipitated protein (Figure 8, B and C). Lysates from cells treated with a proteasome inhibitor, MG132, showed the same 2 main bands of 75 and $45 \mathrm{kDa}$ seen in untreated cells. These bands were evident in both whole-cell lysates immunoblotted with an anti-HA antibody (Figure 8C) and lysates immunoprecipitated with an anti-HA antibody and then probed with the same anti-HA antibody (Figure 7C). The abundance of WT MKRN3 and all MKRN3 mutants was increased after 6 hours of MG132 treatment, with high molecular weight forms greatly enhanced (Figure 8C). The smear evident following MG132 treatment was decreased for all MKRN3 mutants compared with WT MKRN3, with a greater decrease for the MKRN3 mutations located in the RING finger domain, p.C340G and p.R365S (Figure 8C), indicating that these mutations are deleterious to MKRN3-dependent ubiquitination.

\section{Discussion}

The identification of a novel and to date the most common gene associated with CPP, MKRN3, brought a new player to the neuroendocrine regulation of puberty initiation in humans (1). Although previous human genetic studies had demonstrated the crucial role of 2 key stimulators of GnRH secretion, kisspeptin and NKB (27-29), in the excitatory control of puberty, MKRN3 was the first factor to be identified with mutations in humans that likely plays an inhibitory role in GnRH secretion in humans.
MKRN3 is expressed in hypothalamic regions critical for puberty initiation and reproduction in rodents and nonhuman primates, and its temporal pattern of expression in the hypothalamus of mice, rats, and monkeys suggests that MKRN3 actions are more prominent early in life, before the onset of puberty, when its expression levels are highest (1). The decrease in MKRN3 expression before sexual maturation in the ARC and AVPV supports the model of MKRN3 as a component of the inhibitory network suppressing the HPG axis before puberty initiation and is in agreement with the findings of loss-of-function mutations in MKRN3 in patients with precocious re-activation of the axis.

Having established that hypothalamic expression of MKRN3 decreases in both rodents and nonhuman primates before puberty initiation, we set out to investigate if the prepubertal decrease in $M K R N 3$ expression might be the consequence of changes in gonadal steroid levels during sexual development. We excluded this mechanism of regulation of MKRN3 based on (i) the similar Mkrn3 mRNA levels detected in the MBH and POA of WT and $h p g$ mice, which lack GnRH and, therefore, are unable to sexually mature and have low levels of sex steroids (14); and (ii) the lack of effect of estrogen on $M k r n 3$ expression in these 2 regions of the brain. These results are not entirely unexpected because the decrease in hypothalamic $M k r n 3$ expression occurs before any measurable pubertal increase in sex steroid production. Recently, the microRNA, miR30, was shown to operate as a repressor of MKRN3 in the hypothalamus (30).

Estradiol has been shown to differentially regulate Kiss1 mRNA expression in the mouse ARC and AVPV (23). While estradiol inhibits Kiss 1 expression in the ARC, it exerts the opposite effect in the AVPV (31). Our results confirm these findings and argue against a role of MKRN3 in the differential regulation of Kiss1 expression in the ARC and AVPV by estradiol. Altogether, these results demonstrate that Mkrn3 expression in the prepubertal hypothalamus is independent of gonadal activation, consistent with a role as a central regulator of pubertal timing. Using ISH, we found that Mkrn3 mRNA is expressed in several regions of the brain at P10 in addition to the ARC and AVPV. One of the areas where Mkrn3 expression is particularly abundant is the VMN, a hypothalamic nucleus known to be important for the regulation of food intake and energy expenditure $(32,33)$. Although MKRN3 is one of the genes located in the PWS critical region, its contributions to this syndrome are not clear. The expression of $M k r n 3$ in 2 nuclei important for appetite regulation, ARC and VMN, suggests 
A

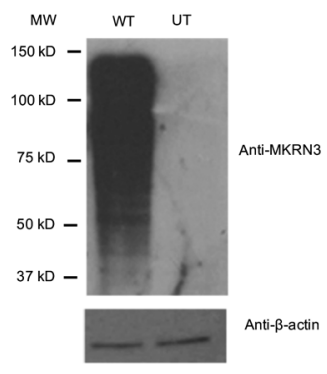

B

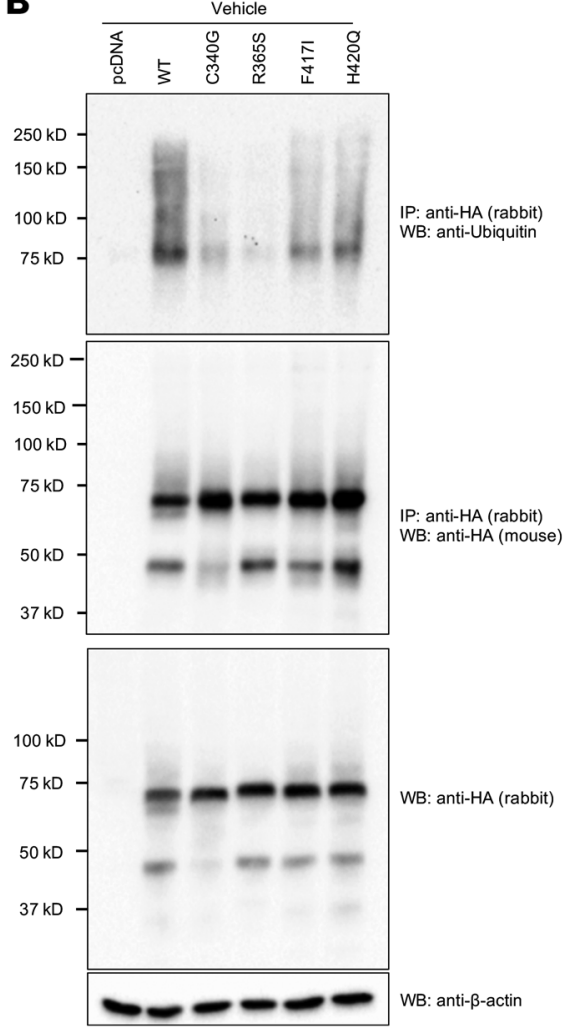

C

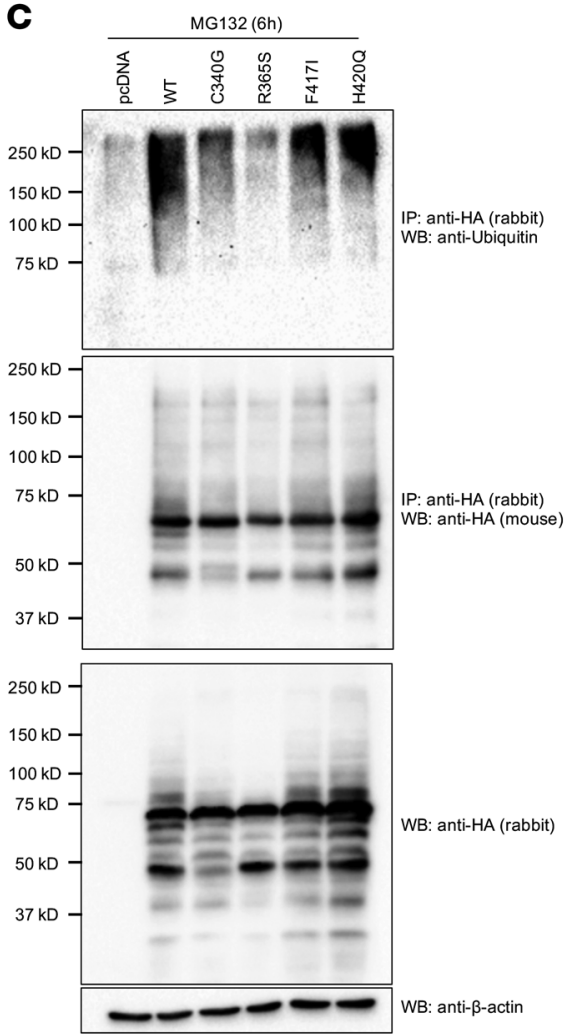

Figure 8. MKRN3 undergoes auto-ubiquitination and MKRN3 mutants are less ubiquitinated than WT MKRN3. (A) HEK293T cells were transfected with a plasmid encoding WT MKRN3 (WT), or were untransfected (UT). The membrane was immunoblotted with anti-MKRN3, and with anti- $\beta$-actin as control. Whole-cell lysates revealed a smear, typical of the pattern shown by polyubiquitinated proteins. Coexpression of his-ubiquitin with MKRN3 resulted in a smear of larger complexes, corresponding to polyubiquitinated protein. (B and C) HEK293T cells were transfected with empty pcDNA, WT MKRN3, or mutant MKRN3. Immune complexes were precipitated using protein A anti-HA beads and probed with anti-ubiquitin (upper panel) or anti-HA (middle panel); lower panels correspond to whole-cell lysates probed with anti-HA. (B) Transfected cells treated with vehicle. Upper panel: An upper smear is present when immunoblotted with anti-ubiquitin; it is stronger in the lane loaded with protein from cells transfected with WT MKRN3 than with MKRN3 mutants. Middle panel: Immunoblotting with anti-HA shows a main band below $75 \mathrm{kDa}$ in lanes with WT MKRN3 and MKRN3 mutants, likely corresponding to a mature form of MKRN3; a band at $50 \mathrm{kDa}$ was also detected in lysates from both WT and mutant MRKN3, but was significantly weaker in intensity for C340G MKRN3 compared with WT MKRN3 and other mutants. (C) Transfected cells treated with MG132. Upper panel: Probing with anti-ubiquitin results in a smear suggestive of auto-ubiquitination that is stronger with WT MKRN3 compared with the MKRN3 mutants located in the zinc finger, F417I and H420G, and is weakest for the RING finger mutants, C340G and R365S. Middle panel: Probed with anti-HA, protein levels are similar for WT MKRN3 and all mutants. In all, anti- $\beta$-actin antibody (bottom panels) was a loading control. WT, WT MKRN3; UT, untransfected cells; MW, molecular weight.

a possible role in the regulation of metabolism, which has not yet been explored. To date, patients with CPP and loss-of-function mutations in MKRN3 have not been reported to have obesity or other phenotypes (10). The higher levels of Mkrn3 expression in the hypothalamus compared with other tissues early in life underscore the importance of MKRN3 in this region. The decrease in expression in the hypothalamus and cortex during development is consistent with a possible role in neurodevelopment. Indeed, the makorin gene family is abundantly expressed in the developing brain and nervous system and one of the members of this family, MKRN2, has been shown to play a role in neurogenesis $(11,34)$. Our results show that, in contrast to the brain, $M k r n 3$ expression does not decrease in peripheral tissues such as the liver and testes. Mkrn3 expression in these tissues is low at P9 and remains low at P90, which may help to explain why patients with loss-of-function mutation in MKRN3 present with isolated CPP but no other detectable clinical manifestations. Further follow-up of patients with known loss-of-function mutations in MKRN3 will help to clarify the role of MKRN3 in brain development and other tissues. Moreover, it would be very informative to measure the expression of MKRN3 in human hypothalamus.

In this study, we investigated possible mechanisms of action by which MKRN3 may influence pubertal timing. As noted earlier, kisspeptin and NKB have been shown to be important players in the regulation of GnRH secretion $(29,35-37)$ and to act in synchrony to regulate GnRH secretion (35). In fact, Kiss1 mRNA-expressing neurons in the ARC also express Tac3 mRNA (13). Compelling evidence suggests that the stimulatory role of NKB in GnRH release in a number of species is in large part mediated by kisspeptin $(35,38,39)$. Hypothalamic levels of kisspeptin have been shown to increase across postnatal development $(40,41)$, suggesting that kisspeptin in the hypothalamus is upregulated around the time of puberty. Similarly, Tac3 expression also increases during pubertal development in the mouse ARC, with the time course of the increase in striking parallel to the decrease in Mkrn3 expression $(1,42)$. Therefore, we hypothesized that MKRN3 might act in 
the ARC to inhibit KISS1 and/or TAC3 gene activity. Using ISH and single-cell RT-PCR, we showed that Mkrn3 is indeed expressed in Kiss1 neurons of the mouse ARC.

Given the inverse correlation of Mkrn3 expression with Kiss1 and Tac3 expression in the ARC, combined with the colocalization of Mkrn3 expression in ARC Kiss1 neurons by ISH, we proposed that one possible mechanism by which MKRN3 might inhibit GnRH secretion is through a repressive effect on KISS1 and/ or TAC3 gene transcription. We tested this hypothesis through a series of luciferase assays using an in vitro cell model. These studies showed that MKRN3 inhibited KISS1 and TAC3 promoter activity, without affecting the promoter activity of other genes implicated in either the stimulatory (EAP1, Ttf1, and Vglut2) or the inhibitory (Viaat, Eed, and PDYN) control of puberty. These findings suggest that MKRN3 is acting selectively within Kiss1 neurons to inhibit the expression of KISS1 and TAC3, which encode neuropeptides that are central to the stimulatory control of $\mathrm{GnRH}$ secretion. Notably, MKRN3 did not alter the promoter activity of $P D Y N$, a gene encoding the inhibitory peptide dynorphin, which is coexpressed with KISS1 and TAC3 in ARC Kiss1 neurons.

Additional assays testing MKRN3 variants carrying missense mutations associated with CPP showed that mutations p.R365S and p.C340G, located in the RING finger domain of MKRN3, compromised the ability of MKRN3 to inhibit both KISS1 and TAC3 promoter activity. The loss of repressive activity was more pronounced for the p.C340G mutant, likely because loss of cysteine at position 340 prevents the zinc-dependent structural organization of the RING finger domain (23). MKRN3 proteins carrying p.F417I and p.H420Q mutations of the C3H zinc finger near the $\mathrm{C}$-terminus were as effective as WT MKRN3 in repressing the TAC3 promoter, but exhibited partial loss of activity on the KISS1 promoter. These findings suggest that while integrity of the RING finger domain is essential for MKRN3 to repress both KISS1 and TAC3 promoter activity, the C-terminal zinc domain is not essential for MKRN3 repression of the TAC3 promoter. Because zinc finger domain-mediated interactions are a hallmark of repressive complexes formed by zinc finger domain-containing proteins (43), it is possible that in the absence of a functional C-terminal zinc finger domain, the 2 upstream domains present in MKRN3 suffice to ensure the occurrence of meaningful protein-protein interactions leading to gene repression.

Structural analysis by $\mathrm{x}$-ray crystallography and in silico structural models showed that phenylalanine 417 is located close to the zinc binding site and is likely important for MKRN3 function (22). These studies also indicated that the mutation p.H420Q likely alters the conformation of the zinc finger, because $\mathrm{Q} 420$ is predicted to be more distant from the zinc ion than H420, thereby likely interfering with the zinc finger conformation (22). As noted above, all mutations tested here were identified only in patients with CPP and their respective fathers, following the expected inheritance pattern for a maternally imprinted gene, indicating that these mutations are the cause of the phenotype in these patients. These considerations suggest that the $\mathrm{C}$-terminal zinc finger mutations may cause CPP by impairing the ability of MKRN3 to repress puberty-stimulatory genes other than KISS1 and TAC3.

Notably, ChIP assays showed that WT MKRN3 as well as the p.C340G, p.R365S, and p.H420Q mutants all bound equally well to the KISS1 and TAC3 promoters, suggesting that MKRN3 recruitment to these promoters does not require an intact, functional RING finger domain. This supports the hypothesis that MKRN3 is not acting directly as a transcriptional regulator of these genes, but rather acting indirectly, possibly as part of a transcriptional network to repress KISS1 and TAC3 promoter activity Although the p.F417I MKRN3 mutant had lower binding to the KISS1 and TAC3 promoters in ChIP assays, this mutant was still capable of inhibiting the promoters in vitro. As discussed above, it is likely that 2 intact upstream zinc finger motifs are able to ensure sufficient binding to mediate transcriptional repression of these gene promoters.

Indeed, the presence of the RING finger domain is perhaps the most salient structural feature of MKRN3, not only because this region is a feature of proteins forming repressive complexes to diminish target gene activity (44), but also because it is a signature domain of E3 ubiquitin ligases (45). MKRN3 has also been shown to be an E3 ubiquitin ligase (46). To date, 22 MKRN3 missense mutations in different domains of MKRN3 have been associated with CPP. Most of them are located in the RING finger domain, highlighting the importance of this domain for the protein function (10). Our Western blot analysis demonstrated that MKRN3 has ubiquitin ligase activity, as assessed by the ability of MKRN3 to become auto-ubiquitinated, a feature characteristic of E3 ubiquitin ligases (26). This ubiquitination was diminished in the MKRN3 variants tested, and more markedly for those carrying missense mutations in the RING finger domain (p.C340G and p.R365S MKRN3).

The finding that the RING finger domain is important for both MKRN3 trans-inhibitory activity and ubiquitination raises the possibility that the mechanism underlying MKRN3-dependent inhibition of KISS1 and TAC3 transcription involves ubiquitination of molecules required for efficient repression of these genes, as proposed above. An example of such a mechanism is ubiquination of histone $2 \mathrm{~A}$ at lysine 119, a necessary step in the sequence of events underlying polycomb-dependent repression of gene transcription (47), and another example is the ubiquitination of H2AK119 for transcriptional repression (48). Because ubiquitination is frequently associated with protein degradation (45), it is possible that MKRN3 represses KISS1 and TAC3 transcription by ubiquitinating proteins associated with the transcriptional machinery that specifically regulate KISS1 and TAC3 expression. Future studies are necessary to address these possibilities.

One-third (36\%) of the MKRN3 mutations identified in patients with CPP are frameshift mutations located at alanine 162 , resulting in a truncated protein lacking the RING and 2 zinc finger domains (10). Our results show that a truncated form of MKRN3 similar to the frameshift identified in several patients, lacking the RING finger and 2 zinc fingers domains, was unable to repress KISS1 and TAC3 promoter activity. Given the complex structure of MKRN3 with several important motifs, it is likely that these frameshift mutations result in loss of function through several mechanisms.

In summary, this work presents data that support a role for MKRN3 in the central control of the timing of pubertal initiation and GnRH release through the inhibition of KISS1 and TAC3 transcription in Kiss1 neurons in the hypothalamus, at least in part through mechanisms involving the RING finger domain. These findings are 
in agreement with human genetic studies, which revealed that the loss of MKRN3 results in central precocious puberty.

\section{Methods}

Western blotting, luciferase studies, gene expression profiles, and RT-qPCR experiments were all repeated at least 3 times.

\section{Cell culture}

HEK293T (ATCC CRL-3216) and Neuro-2a (ATCC CCL-131) cells were grown and maintained in DMEM supplemented with 10\% (vol/ vol) fetal bovine serum (Omega), $100 \mathrm{U} / \mathrm{mL}$ penicillin, and $100 \mu \mathrm{g} / \mathrm{mL}$ streptomycin sulfate (Invitrogen) in $5 \% \mathrm{CO}_{2}$ humidified air at $37^{\circ} \mathrm{C}$.

\section{Expression vectors}

A pReceiver plasmid with a CMV promoter and $3 \times$ HA tag encoding MKRN3 was purchased from GeneCopoeia (EX-F0470-M06). Mutations were introduced into the WT vector using the QuikChange Site-Directed Mutagenesis kit (Stratagene) according to the manufacturer's protocol. Plasmids were prepared using maxiprep kits (QIAGEN). Mutations were confirmed by bidirectional sequencing at the Dana-Farber/Harvard Cancer Center DNA Resource Core (Boston, Massachusetts, USA). Sequences were checked using Sequencher 5.4.6 (Gene Code Corporation). A pcDNA3 plasmid encoding ubiquitin was purchased from Addgene (plasmid 18712).

Gene promoters were cloned into the pGL2-luciferase vector (Promega) as described previously (ref. 49 and Supplemental Table 2).

\section{Western blot analysis and immunoprecipitation}

HEK293T cells were plated in 12-well plates and transiently transfected with pReceiver plasmid with a CMV promoter encoding either WT or mutant MKRN3, and in some cases cotransfected with a ubiquitin plasmid using Lipofectamine 2000 following the manufacturer's protocol (Invitrogen). Twenty-four hours after transfection, total cellular proteins were extracted using M-PER mammalian protein extract buffer (Thermo Fisher Scientific). Ten micrograms of soluble protein per lane was resolved by immunoblot. The blots were then incubated with anti-MKRN3 antibody (Abcam ab140267, 1:3000, polyclonal), anti-HA antibody (Abcam ab9110, 1:4000, polyclonal), or anti-ubiquitin antibody (BioLegend 646301, 1:1000, P4D1) overnight at $4^{\circ} \mathrm{C}$. Blots were incubated with anti-rabbit secondary antibodies linked to horseradish peroxidase (Bio-Rad 170-6515, 1:5000-1:10000); the antibody-antigen complexes were visualized using an enhanced chemiluminescence reagent (PerkinElmer). Blots were stripped using stripping buffer (Thermo Fisher Scientific) and reprobed with anti- $\beta$ actin antibody (MilliporeSigma A3854, 1:10,000, AC-15).

For immunoprecipitation, HEK293T cells were plated and WT or mutant MKRN3 constructs were transfected for 24 hours. Cells were treated with vehicle or MG132 (10 $\mu \mathrm{M}$ in DMSO solution, Invitrogen) for 6 hours, and then cells were lysed using the M-PER lysis buffer (Thermo Fisher Scientific). Ten percent of proteins was used for total protein samples. After preclearing of the proteins with protein $A / G^{-}$ agarose beads (Invitrogen) for 30 minutes, supernatants were incubated with anti-HA (Abcam ab9110) for at least 3 hours at $4^{\circ} \mathrm{C}$. Immune complexes were precipitated using protein A beads overnight at $4^{\circ} \mathrm{C}$ and washed 3 to 5 times before elution with $5 \times$ SDS-PAGE sample buffer. Ubiquitin-conjugated MKRN3 was immunoblotted with anti-ubiquitin (BioLegend 646301, 1:1000, P4D1).

\section{Animals}

Mice, rats, and monkeys were maintained in a 12-hour light/12-hour dark cycle, controlled temperature, and fed a standard diet and water ad libitum.

\section{Mice}

Adult WT male and female C57BL/6 mice were purchased from Charles River Laboratories. Female hypogonadal $(h p g)$ mice with the Gnrh1 gene deleted and WT littermates were bred and genotyped as previously described (50).

For single-cell PCR studies, 13 -day-old female Kiss $1^{\text {CreGFP }}$ mice (51) were bred in the colony at Oregon Health and Sciences University (OHSU). Mice were genotyped and used for experiments on day 13.

Mouse tissue collection. For RT-qPCR, intact male and female $h p g$ mice and WT littermates were sacrificed at the stated ages. Brains were removed and coronal sections of brain tissue (1 mm thick) encompassing the regions containing either the ARC or AVPV were obtained with the aid of a 1-mm coronal brain matrix (Braintree Scientific). The ARC and AVPV were then isolated from each section under a dissection microscope by 2 bilateral parasagittal cuts, each $0.5 \mathrm{~mm}$ lateral to the midline, and 1 horizontal cut $1 \mathrm{~mm}$ dorsal of the ventral surface, yielding total tissue sizes of approximately $1 \mathrm{~mm}^{3}$ that were immediately frozen in liquid nitrogen.

The rostral and caudal areas of the hypothalamus, including the POA and the $\mathrm{MBH}$, respectively, were isolated by 2 bilateral parasagittal cuts, each $0.5 \mathrm{~mm}$ lateral to the midline between the optic chiasm and the posterior commissure, and 1 horizontal cut $1 \mathrm{~mm}$ dorsal of the ventral surface. A midline incision was made in the abdomen and the liver was removed. Testes were isolated through a small scrotal incision.

For the single-cell PCR studies, mouse brains were removed and $240-\mu \mathrm{m}$ slices were cut on a vibratome in cold, oxygenated, sucrose-containing artificial cerebrospinal fluid (aCSF [in mM]: 208 sucrose, $2 \mathrm{KCl}, 1 \mathrm{MgCl}_{2}, 1.25 \mathrm{NaH}_{2} \mathrm{PO}_{4}$, 10 HEPES, $26 \mathrm{NaHCO}_{3}, 10$ dextrose, $2 \mathrm{MgSO}_{4}$, and $1 \mathrm{CaCl}_{2}$ ). The slices were transferred to an auxiliary chamber containing oxygenated aCSF for 1.5 to 2 hours (in mM: $124 \mathrm{NaCl}, 5 \mathrm{KCl}, 1.44 \mathrm{NaH}_{2} \mathrm{PO}_{4}, 5$ HEPES, 10 dextrose, 25.99 $\mathrm{NaHCO}_{3}, 2 \mathrm{CaCl}_{2}$ ) until cell dispersion.

\section{Rats}

Immature Sprague-Dawley rats were obtained from Charles River Laboratories.

Rat tissue collection. To measure changes in gene expression that occur in the hypothalamus during prepubertal development, the $\mathrm{MBH}$ of female rats was dissected by a rostral cut along the posterior border of the optic chiasm, a caudal cut immediately in front of the mammillary bodies, and 2 lateral cuts half-way between the median eminence and the hypothalamic sulci. The thickness of the tissue fragment was approximately $2 \mathrm{~mm}$. Upon dissection, the tissues were immediately frozen on dry ice.

\section{Nonhuman primates}

Female rhesus monkeys (Macaca mulatta) were obtained through the Oregon National Primate Research Center (ONPRC) Tissue Distribution Program, and were classified into different stages of development, based on the age of the animals and the pubertal stages reported by Watanabe and Terasawa (52). 


\section{Monkey tissue collection}

The brain was removed from the cranium and the $\mathrm{MBH}$ was dissected as previously described (53), i.e., with a rostral cut along the posterior border of the optic chiasm, a caudal cut immediately anterior to the mammillary bodies, and 2 lateral cuts along the hypothalamic sulci. The tissue was rapidly frozen on dry ice.

\section{RNA extraction}

All samples collected for gene expression analyses were immediately frozen in dry ice. Total RNA was extracted from the collected tissues using TRIzol (Invitrogen) or the RNeasy Mini Kit (QIAGEN), according to the manufacturers' protocols. Total RNA concentration was carefully measured by UV spectrophotometry and RNA integrity was confirmed by gel electrophoresis.

\section{RT-qPCR}

Total RNA (500 ng) was reverse transcribed using the Superscript III cDNA synthesis kit (Invitrogen) as described previously $(49,54)$, followed by quantitative real-time PCR analysis on an ABI Prism 7000 sequence detection system (Applied Biosystems). SYBR Green mix (Bio-Rad) was used according to the manufacturer's instructions. To normalize the mRNA values obtained, Ppia (peptidylprolyl isomerase A) or RpL19 (ribosomal protein L19) mRNA was measured in each sample as a housekeeping gene. Gene accession numbers are described in Supplemental Table 1.

\section{Estrogen treatment}

We treated WT female mice at P11 with $2 \mu \mathrm{g}$ of EB ( $3 \mu \mathrm{g}$ per $10 \mathrm{~g}$ body weight) or vehicle (sesame oil) subcutaneously to determine the effect of EB on MBH and POA Mkrn3 mRNA levels. All mice were sacrificed 24 hours after treatment and the POA and $\mathrm{MBH}$ were removed as described above for RT-qPCR analysis of Mkrn3.

\section{Single-label ISH of Mkrn3 mRNA}

Mkrn3 mRNA sense and antisense probes were transcribed with T7 or T3 polymerase (Fermentas), as described previously (13). Briefly, radio-labeled probes were synthesized in vitro by inclusion of the following ingredients in a volume of $20 \mu \mathrm{L}$ : $250 \mathrm{Ci}$ [ $\left.{ }^{33} \mathrm{P}\right] \mathrm{UTP}$ (PerkinElmer Life and Analytical Sciences), $1 \mu \mathrm{g}$ of PCR product, $0.5 \mathrm{mM}$ each ATP, CTP, and GTP, and $40 \mathrm{U}$ of polymerase. Residual DNA was digested with $4 \mathrm{U}$ of DNase (Ambion), and the DNase reaction was terminated by addition of $2 \mu \mathrm{L}$ of 0.5M EDTA, $\mathrm{pH}$ 8.0. The riboprobes were separated from unincorporated nucleotides with ProbeQuant G-50 Micro Columns (GE Health Care). Slides with brain and hypothalamic sections from the different experimental groups were processed as reported previously (38).

\section{FISH}

The brains from two 12-day-old and two 30-day-old female mice were fixed by intracardiac perfusion of $4 \%$ paraformaldehyde borate buffer, $\mathrm{pH}$ 9.5, and were processed for hybridization histochemistry, as previously described $(55,56)$. For identification of mRNAs expressed in the same cell, we used the double-labeled FISH procedure described by Watakabe et al. (57), with small modifications (54). We employed an rMkrn3 cRNA probe labeled with fluorescein12-UTP (FITC) and an rKiss1 cRNA probe labeled with digoxigenin11-UTP (Dig). The Kiss1 cRNA probe was transcribed from a 379-bp rat Kiss1 cDNA corresponding to nucleotides (nt) 1-379 in rat Kiss1 mRNA (58), and the rMkrn3 probe was generated by in vitro transcription of a 549-bp cDNA template complementary to nt 1381-1930 in the coding region of rat Mkrn3 mRNA (59). cDNAs were subcloned into the pGEM-T vector (Promega). The labeling reactions were performed in a $10 \mu \mathrm{L}$ volume, as reported previously (60). Control sections were incubated with sense probes transcribed from the same plasmid, but linearized at the $3^{\prime}$ end to transcribe the coding strand of the cDNA template.

\section{ChIP assay}

To assess the recruitment of MKRN3 to gene promoters, we performed ChIP assays using chromatin extracted from HEK293T cells transfected with MKRN3-HA constructs (WT and the MKRN3 mutants). The ChIP procedure was described previously $(49,54,61)$, and was carried out with minimal modifications, including the use of a dry-heat shaker (Thermo Fisher Scientific) for both the elution and de-cross-link steps $\left(65^{\circ} \mathrm{C}\right.$ for 1 hour and $95^{\circ} \mathrm{C}$ for 2 hours, respectively).

\section{PCR detection of chromatin immunoprecipitated DNA}

Genomic regions of interest were amplified by PCR. Accession numbers of the genes analyzed as well as the chromosomal position of the 5 ' flanking region amplified, using the position of the transcription start site (TSS) as the reference point, are shown in Supplemental Table 1. The primer sequences (Eurofins MWG Operon) used to detect the DNA fragment of interest in the immunoprecipitated DNA are also shown in Supplemental Table 1. PCR reactions were performed as described previously $(3,4)$. Data are expressed as percentage of IP signal/Input signal. To detect bound MKRN3-HA, we used an anti-HA rabbit antibody (Abcam ab9110) at $2 \mu \mathrm{g} /$ reaction.

\section{Single-cell PCR data analysis}

Cell dispersion and harvesting. The method used for neuronal harvesting was according to previously established procedures, with modifications as outlined below (62). The ARC was microdissected and incubated in protease at $37^{\circ} \mathrm{C}$ for 15 minutes. Gentle trituration with glass polished Pasteur pipets was used to disperse the neurons on a glass-bottom dish. A constant flow of oxygenated aCSF bathed the neurons, flushing out debris and dead cells. Healthy neurons with smooth membranes and intact processes were visualized using a Leitz inverted microscope, patched, and then harvested with gentle suction using the XenoWorks microinjector system (Sutter Instruments). The single cells were expelled into a siliconized microcentrifuge tube containing $1 \mu \mathrm{L}$ Invitrogen Superscript III $5 \times$ Buffer, 15 $\mathrm{U}$ of RNasin (Promega), and $10 \mathrm{mM}$ dithiothreitol in a total volume of $5 \mu \mathrm{L}$. Single cells were treated with DNase I (RNase-free DNase I, Invitrogen). The DNAse I ( $2 \mathrm{U} / \mu \mathrm{L})$ was diluted 1:10,000 and added to each tube, incubated at $37^{\circ} \mathrm{C}$ for 5 minutes, and then denatured at $65^{\circ} \mathrm{C}$ for 5 minutes. Each harvested cell was then reverse transcribed as described previously (62) with modifications in which both random primers (100 ng/cell, Promega) and anchored oligo(dT) ${ }_{20}$ primer (400 ng/cell, Invitrogen), and Superscript III reverse transcriptase (100 U/per cell, Invitrogen) were used. Harvested aCSF samples in the vicinity of the dispersed cells also underwent RT and were used as control. Cells and tissue RNA used as negative controls were processed as described above but without reverse transcriptase. Each cell was then evaluated using PCR. 
Single-cell RT-PCR. The PCR was performed using 3 to $4 \mu \mathrm{L}$ of cDNA template from each RT reaction in a $30-\mu \mathrm{L}$ PCR mix. Fifty cycles of amplification were performed using a Bio-Rad C1000 Thermal Cycler as follows: initial denaturation at $94^{\circ} \mathrm{C}$ for 2 minutes, denaturation at $94^{\circ} \mathrm{C}$ for 20 seconds, annealing at $57^{\circ} \mathrm{C}$ for 30 seconds (Kiss1) or at $60^{\circ} \mathrm{C}$ for 30 seconds $(M k r n 3)$, extension at $72^{\circ} \mathrm{C}$ for 30 seconds, with a final extension at $72^{\circ} \mathrm{C}$ for 5 minutes (62). PCR products were visualized with ethidium bromide in a $2 \%$ agarose gel.

\section{Functional promoter assays}

Neuro-2a cells were transfected with luciferase reporter constructs (12) containing the $5^{\prime}$ flanking region of the putative target genes, in addition to WT MKRN3-HA or the MKRN3 mutants. Cells were cultured as described previously $(3,4)$. Twenty-four hours later, the reporter constructs (in the luciferase reporter plasmid pGL2) were transiently cotransfected along with WT or mutant MKRN3-HA for 5 hours using Lipofectamine 2000 (Invitrogen) at a ratio of $1 \mu \mathrm{g}$ DNA/2.5 $\mu \mathrm{L}$ Lipofectamine 2000 in Optimem (Invitrogen). After transfection, the cells were returned to serum-containing DMEM medium. Forty-eight hours later, they were harvested and assayed for luciferase activity using the Firefly Luciferase Glow Assay Kit (Pierce). Transfection efficiency was normalized by cotransfecting the plasmid CMV-Sport-beta-gal (Invitrogen) at $10 \mathrm{ng} / \mathrm{mL}$ and determining $\beta$-galactosidase activity using the Tropix Galacto Light Plus (ABI), as reported previously (63).

\section{Experimental identification of MKRN3 variants by $3^{\prime}$-RACE PCR}

To determine the existence of MKRN3 3' variants in human hypothalamic RNA, we used total RNA extracted from adult human hypothalamus. The donor was a 23-year-old White male who died of cardiac arrest (Ambion 6864). We used the switching mechanism at the $3^{\prime}$ end of RNA transcript rapid amplification of cDNA ends (SMART RACE cDNA Amplification kit, Clontech Laboratories) according to the manufacturer's recommendations, as described previously, with minimal modifications (64). In brief, $1 \mu \mathrm{g}$ of total RNA from human hypothalamus was reverse transcribed using a modified oligo(dT) primer, SMARTScribe Reverse Transcriptase, and the SMARTer II A Oligonucleotide, which anneals to the extended cDNA tail added by the SMARTScribe Reverse Transcriptase to the 3 ' end of the first-strand cDNA. The resulting cDNAs were amplified by 2 rounds of PCR using HotStart Taq polymerase (QIAGEN). The first round of PCR was performed with a gene-specific forward primer (GSFP) inside the MKRN3 coding region (5'-GCCTCAAGCCCATAAAGAAAAA-3') and the kit's AP1 primer. The second nested PCR was performed using $1 / 100$ of the initial PCR product and amplifying with the GSFP and the kit's AP2 primer (Supplemental Figure 2). The PCR products were subcloned into the plasmid pGEM-T (Promega), and some of the resulting colonies were sequenced from both ends using M13 forward and M13 reverse primers.

\section{Statistics}

All data are expressed as the mean \pm SEM for each group. For experiments with 2 comparisons, 2-tailed Student's $t$ test was used for statistical analysis. For experiments with multiple comparisons, the level of significance was determined using ANOVA followed by post hoc tests as indicated in the results. Significance level was set at $P$ less than 0.05. All analyses were performed with GraphPad Prism software.

For determination of single-neuron expression of a particular transcript in single-cell PCR experiments, the number of GFP-positive cells expressing each transcript was counted for each animal and percentage expression determined compared with Kiss $1^{\mathrm{GFP}}$-expressing neurons in each animal.

\section{Study approval}

The study was approved by the Medical Ethics Committee of Brigham and Women's Hospital and the ONPRC. All mouse studies were approved by the IACUC of Brigham and Women's Hospital and of OHSU, by the Harvard Medical Area Standing Committee on Animals in the Harvard Medical School Center for Animal Resources and Comparative Medicine. The use of rats and nonhuman primates was approved by the ONPRC Animal Care and Use Committee. All animal experiments were carried out in accordance with the NIH Guide for the Care and Use of Laboratory Animals (National Academies Press, 2011) and abided by the declaration of ethical approval for experiments.

\section{Author contributions}

APA and AL performed most of the experiments with the assistance from CAT, JNL, YBS, AE, and CFA. APA, AL, ACL, SO, and UBK designed the experiments. VMN performed the ISH. MAB and OKR carried out the single-cell RT-qPCR experiments. APA and $\mathrm{AL}$ analyzed the data. APA, AL, SO, and UBK wrote the manuscript. RSC, SO, and UBK supervised the research data. APA, UBK, and SO conceived the research study. The manuscript was reviewed and edited by all authors.

\section{Acknowledgments}

This work was supported by grants from the Eunice Kennedy Shriver National Institute of Child Health and Human Development (R00 HD091381 to APA and R01 HD082314 to UBK) and the NIH (R01 HD084542 to AL and SO, R01 DK068098 to OKR, and P51 OD011092 for the operation of the Oregon National Primate Research Center). The authors would like to thank Han K. Kim and Melissa Magnuson for their assistance with the project and Victor Vaz for his assistance with the graphical abstract.

Address correspondence to: Ana Paula Abreu, Division of Endocrinology, Diabetes, and Hypertension, Brigham and Women's Hospital, 221 Longwood Avenue, Boston, Massachusetts 02115, USA. Phone: 617.278.0405; Email: apabreu@bwh.harvard.edu.

\footnotetext{
1. Abreu AP, et al. Central precocious puberty caused by mutations in the imprinted gene MKRN3. N Engl J Med. 2013;368(26):2467-2475.

2. Jong MT, et al. Imprinting of a RING zinc-finger encoding gene in the mouse chromosome region homologous to the Prader-Willi syndrome genetic region. Hum Mol Genet. 1999;8(5):795-803.
}

\footnotetext{
3. Jong MT, et al. A novel imprinted gene, encoding a RING zinc-finger protein, and overlapping antisense transcript in the Prader-Willi syndrome critical region. Hum Mol Genet. 1999;8(5):783-793.

4. Abreu AP, Macedo DB, Brito VN, Kaiser UB, Latronico AC. A new pathway in the control of the initiation of puberty: the MKRN3 gene. JMol
}

Endocrinol. 2015;54(3):R131-R139.

5. Abreu AP, Kaiser UB. Pubertal development and regulation. Lancet Diabetes Endocrinol. 2016;4(3):254-264.

6. Terasawa E, Fernandez DL. Neurobiological mechanisms of the onset of puberty in primates. Endocr Rev. 2001;22(1):111-151. 
7. Palmert MR, Boepple PA. Variation in the timing of puberty: clinical spectrum and genetic investigation. JClin Endocrinol Metab. 2001;86(6):2364-2368.

8. Rosenfield RL, Lipton RB, Drum ML. Thelarche, pubarche, and menarche attainment in children with normal and elevated body mass index. Pediatrics. 2009;123(1):84-88.

9. Gajdos ZK, Hirschhorn JN, Palmert MR. What controls the timing of puberty? An update on progress from genetic investigation. Curr Opin Endocrinol Diabetes Obes. 2009;16(1):16-24.

10. Valadares LP, et al. MKRN3 Mutations in central precocious puberty: a systematic review and meta-analysis. J Endocr Soc. 2019;3(5):979-995.

11. Gray TA, et al. The ancient source of a distinct gene family encoding proteins featuring RING and $\mathrm{C}(3) \mathrm{H}$ zinc-finger motifs with abundant expression in developing brain and nervous system. Genomics. 2000;66(1):76-86.

12. Hughes IA. Releasing the brake on puberty. N Engl JMed. 2013;368(26):2513-2515.

13. Navarro VM, Gottsch ML, Chavkin C, Okamura H, Clifton DK, Steiner RA. Regulation of gonadotropin-releasing hormone secretion by kisspeptin/dynorphin/neurokinin B neurons in the arcuate nucleus of the mouse. JNeurosci. 2009;29(38):11859-11866.

14. Mason AJ, et al. A deletion truncating the gonadotropin-releasing hormone gene is responsible for hypogonadism in the hpg mouse. Science. 1986;234(4782):1366-1371.

15. Adachi S, et al. Involvement of anteroventral periventricular metastin/kisspeptin neurons in estrogen positive feedback action on luteinizing hormone release in female rats. J Reprod Dev. 2007;53(2):367-378.

16. Smith JT, Cunningham MJ, Rissman EF, Clifton DK, Steiner RA. Regulation of Kiss1 gene expression in the brain of the female mouse. Endocrinology. 2005;146(9):3686-3692.

17. Goodman RL, et al. Kisspeptin neurons in the arcuate nucleus of the ewe express both dynorphin A and neurokinin B. Endocrinology. 2007;148(12):5752-5760.

18. Hrabovszky E, et al. The kisspeptin system of the human hypothalamus: sexual dimorphism and relationship with gonadotropin-releasing hormone and neurokinin B neurons. Eur J Neurosci. 2010;31(11):1984-1998.

19. Ramaswamy S, Seminara SB, Ali B, Ciofi P, Amin NA, Plant TM. Neurokinin B stimulates GnRH release in the male monkey (Macaca mulatta) and is colocalized with kisspeptin in the arcuate nucleus. Endocrinology. 2010;151(9):4494-4503.

20. Toro CA, Wright H, Aylwin CF, Ojeda SR, Lomniczi A. Trithorax dependent changes in chromatin landscape at enhancer and promoter regions drive female puberty. Nat Commun. 2018;9(1):57.

21. Mancini A, et al. EAP1 regulation of GnRH promoter activity is important for human pubertal timing. Hum Mol Genet. 2019;28(8):1357-1368.

22. de Vries L, Gat-Yablonski G, Dror N, Singer A, Phillip M. A novel MKRN3 missense mutation causing familial precocious puberty. Hum Reprod. 2014;29(12):2838-2843.

23. Settas N, Dacou-Voutetakis C, Karantza M, Kanaka-Gantenbein C, Chrousos GP, Voutetakis
A. Central precocious puberty in a girl and early puberty in her brother caused by a novel mutation in the MKRN3 gene. JClin Endocrinol Metab. 2014;99(4):E647-E651.

24. Macedo DB, et al. Central precocious puberty that appears to be sporadic caused by paternally inherited mutations in the imprinted gene makorin ring finger 3. J Clin Endocrinol Metab. 2014;99(6):E1097-E1103.

25. de Bie P, Ciechanover A. Ubiquitination of E3 ligases: self-regulation of the ubiquitin system via proteolytic and non-proteolytic mechanisms. Cell Death Differ. 2011;18(9):1393-1402.

26. Amemiya Y, Azmi P, Seth A. Autoubiquitination of BCA2 RING E3 ligase regulates its own stability and affects cell migration. Mol Cancer Res. 2008;6(9):1385-1396.

27. de Roux N, Genin E, Carel JC, Matsuda F, Chaussain JL, Milgrom E. Hypogonadotropic hypogonadism due to loss of function of the KiSS1-derived peptide receptor GPR54. Proc Natl Acad Sci U S A. 2003;100(19):10972-10976.

28. Seminara SB, et al. The GPR54 gene as a regulator of puberty. N Engl J Med.2003;349(17):1614-1627.

29. Topaloglu AK, et al. TAC3 and TACR3 mutations in familial hypogonadotropic hypogonadism reveal a key role for neurokinin $\mathrm{B}$ in the central control of reproduction. Nat Genet. 2009;41(3):354-358.

30. Heras V, et al. Hypothalamic miR-30 regulates puberty onset via repression of the puberty-suppressing factor, Mkrn3. PLoS Biol. 2019;17(11):e3000532.

31. Herbison AE. Estrogen positive feedback to gonadotropin-releasing hormone ( $\mathrm{GnRH})$ neurons in the rodent: the case for the rostral periventricular area of the third ventricle (RP3V). Brain Res Rev. 2008;57(2):277-287.

32. Schwartz MW, Woods SC, Porte D, Seeley RJ, Baskin DG. Central nervous system control of food intake. Nature. 2000;404(6778):661-671

33. Kalra SP, Dube MG, Pu S, Xu B, Horvath TL, Kalra PS. Interacting appetite-regulating pathways in the hypothalamic regulation of body weight. Endocr Rev. 1999;20(1):68-100.

34. Cheung WK, et al. Identification of protein domains required for makorin-2-mediated neurogenesis inhibition in Xenopus embryos. Biochem Biophys Res Commun. 2010;394(1):18-23.

35. Navarro VM, et al. The integrated hypothalamic tachykinin-kisspeptin system as a central coordinator for reproduction. Endocrinology. 2015;156(2):627-637.

36. Topaloglu AK, Semple RK. Neurokinin B signalling in the human reproductive axis. Mol Cell Endocrinol. 2011;346(1-2):57-64.

37. Qiu J, et al. High-frequency stimulation-induced peptide release synchronizes arcuate kisspeptin neurons and excites GnRH neurons. Elife. 2016;5:e16246.

38. Navarro VM, et al. Interactions between kisspeptin and neurokinin B in the control of $\mathrm{GnRH}$ secretion in the female rat. Am J Physiol Endocrinol Metab. 2011;300(1):E202-E210.

39. Navarro VM. Interactions between kisspeptins and neurokinin B. Adv Exp Med Biol. 2013;784:325-347.

40. Navarro VM, et al. Developmental and hor- monally regulated messenger ribonucleic acid expression of KiSS-1 and its putative receptor, GPR54, in rat hypothalamus and potent luteinizing hormone-releasing activity of KiSS-1 peptide. Endocrinology. 2004;145(10):4565-4574.

41. Shahab M, Mastronardi C, Seminara SB, Crowley WF, Ojeda SR, Plant TM. Increased hypothalamic GPR54 signaling: a potential mechanism for initiation of puberty in primates. Proc Natl Acad Sci U S A. 2005;102(6):2129-2134.

42. Gill JC, et al. Increased neurokinin B (Tac2) expression in the mouse arcuate nucleus is an early marker of pubertal onset with differential sensitivity to sex steroid-negative feedback than Kiss1. Endocrinology. 2012;153(10):4883-4893.

43. Yeung KT, et al. A novel transcription complex that selectively modulates apoptosis of breast cancer cells through regulation of FASTKD2. Mol Cell Biol. 2011;31(11):2287-2298.

44. Childs KS, Goodbourn S. Identification of novel co-repressor molecules for interferon regulatory factor-2. Nucleic Acids Res. 2003;31(12):3016-3026.

45. Deshaies RJ, Joazeiro CA. RING domain E3 ubiquitin ligases. Annu Rev Biochem. 2009;78:399-434.

46. Lee MS, et al. Loss of the E3 ubiquitin ligase MKRN1 represses diet-induced metabolic syndrome through AMPK activation. Nat Commun. 2018;9(1):3404.

47. Wang $\mathrm{H}$, et al. Role of histone $\mathrm{H} 2 \mathrm{~A}$ ubiquitination in Polycomb silencing. Nature. 2004;431(7010):873-878.

48. Tamburri S, et al. Histone H2AK119 mono-ubiquitination is essential for Polycomb-mediated transcriptional repression. Mol Cell. 2020;77(4):840-856.e5.

49. Lomniczi A, et al. Epigenetic regulation of puberty via Zinc finger protein-mediated transcriptional repression. Nat Commun. 2015;6:10195.

50. Gill JC, Wang O, Kakar S, Martinelli E, Carroll RS, Kaiser UB. Reproductive hormonedependent and -independent contributions to developmental changes in kisspeptin in GnRH-deficient hypogonadal mice. PLoS ONE. 2010;5(7):e11911.

51. Gottsch ML, et al. Molecular properties of Kiss1 neurons in the arcuate nucleus of the mouse. Endocrinology. 2011;152(11):4298-4309.

52. Watanabe G, Terasawa E. In vivo release of luteinizing hormone releasing hormone increases with puberty in the female rhesus monkey. Endocrinology. 1989;125(1):92-99.

53. Heger S, et al. Enhanced at puberty 1 (EAP1) is a new transcriptional regulator of the female neuroendocrine reproductive axis. JClin Invest. 2007;117(8):2145-2154.

54. Lomniczi A, et al. Epigenetic control of female puberty. Nat Neurosci. 2013;16(3):281-289.

55. Lee BJ, et al. TTF-1, a homeodomain gene required for diencephalic morphogenesis, is postnatally expressed in the neuroendocrine brain in a developmentally regulated and cell-specific fashion. Mol Cell Neurosci. 2001;17(1):107-126.

56. Rage F, Lee BJ, Ma YJ, Ojeda SR. Estradiol enhances prostaglandin $\mathrm{E} 2$ receptor gene expression in luteinizing hormone-releasing hormone (LHRH) neurons and facilitates the LHRH response to PGE2 by activating a glia-to-neuron signaling pathway. JNeurosci. 1997;17(23):9145-9156. 
57. Watakabe A, Komatsu Y, Ohsawa S, Yamamori T. Fluorescent in situ hybridization technique for cell type identification and characterization in the central nervous system. Methods. 2010;52(4):367-374.

58. Mastronardi C, et al. Deletion of the Ttf1 gene in differentiated neurons disrupts female reproduction without impairing basal ganglia function. $J$ Neurosci. 2006;26(51):13167-13179.

59. Bilger M, Heger S, Brann DW, Paredes A, Ojeda $\mathrm{SR}$. A conditional tetracycline-regulated increase in gamma amino butyric acid production near luteinizing hormone-releasing hormone nerve terminals disrupts estrous cyclicity in the rat. Endocrinology. 2001;142(5):2102-2114.

60. Berg-von der Emde K, et al. Neurotrophins and the neuroendocrine brain: different neurotrophins sustain anatomically and functionally segregated subsets of hypothalamic dopaminergic neurons. J Neurosci. 1995;15(6):4223-4237.

61. Mueller JK, et al. Transcriptional regulation of the human KisS1 gene. Mol Cell Endocrinol. 2011;342(1-2):8-19.

62. Bosch MA, Tonsfeldt KJ, Rønnekleiv OK. mRNA expression of ion channels in GnRH neurons: subtype-specific regulation by $17 \beta$-estradiol. $\mathrm{Mol}$ Cell Endocrinol. 2013;367(1-2):85-97.

63. Lomniczi A, et al. A single-nucleotide polymorphism in the EAP1 gene is associated with amenorrhea/oligomenorrhea in nonhuman primates. Endocrinology. 2012;153(1):339-349.

64. Castellano JM, Wright H, Ojeda SR, Lomniczi A. An alternative transcription start site yields estrogen-unresponsive Kiss1 mRNA transcripts in the hypothalamus of prepubertal female rats. Neuroendocrinology. 2014;99(2):94-107. 